C-A/AP/\#176 October 2004

\title{
Simulations of Bunch Merging in Booster
}

\author{
C.J. Gardner
}

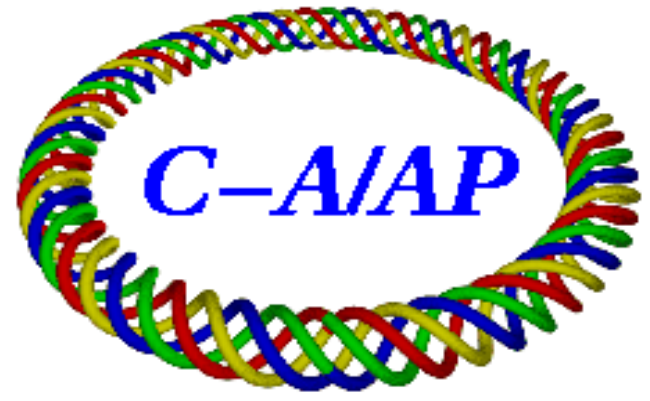

Collider-Accelerator Department Brookhaven National Laboratory Upton, NY 11973 


\title{
Simulations of Bunch Merging in Booster
}

\author{
C.J. Gardner
}

October 13, 2004

Bunch merging schemes developed by J.M. Brennan and the RF group have been used to merge gold and deuteron bunches in Booster for the RHIC physics program. These are described to some extent in Refs. [1] and [2]. Following are notes on simulations of the schemes.

\section{Gold Simulation}

The capture and acceleration of gold ions $\left(\mathrm{Au}^{32+}\right)$ in Booster is discussed in detail in Refs. [3] and [4, 5]. In the simulations described here, unbunched gold ions are first captured at zero bdot and then accelerated up to a flat (zero bdot) porch where the merging takes place. The magnetic field and gap volt programs used are not unlike those used in practice.

\subsection{Magnetic Field Program}

The field $B$ is defined during the time intervals

$$
0<T_{c}<T_{m}<T_{t}<T_{p}<T_{f}
$$

where $T_{c}$ is the capture time, $T_{m}$ is the time at which maximum bdot is reached, $T_{t}$ is the time at which the transition to zero bdot begins, $T_{p}$ is the time at which the merging porch begins, and $T_{f}$ is the final time at the end of the merging process. To obtain a magnetic cycle close to the one used in practice we take

$$
\begin{gathered}
T_{c}=6 \mathrm{~ms}, \quad T_{m}=T_{c}+23 \mathrm{~ms}=29 \mathrm{~ms} \\
T_{t}=T_{m}+5.15 \mathrm{~ms}=34.15 \mathrm{~ms}, \quad T_{p}=T_{t}+11.3 \mathrm{~ms}=45.45 \mathrm{~ms} .
\end{gathered}
$$


The duration $T_{f}-T_{p}$ of the merging process considered here will range from 10 to $40 \mathrm{~ms}$.

Capture takes place at constant field. Thus for $0 \leq t \leq T_{c}$ we have

$$
B(t)=B_{c}, \quad \dot{B}(t)=0, \quad \ddot{B}(t)=0
$$

where $B_{c}=614.71$ Gauss. The dots denote differentiation with respect to time.

For $T_{c} \leq t \leq T_{m}$ we have

$$
\begin{gathered}
B(t)=B_{c}+\dot{B}_{m} \frac{\left(t-T_{c}\right)^{3}}{\left(T_{m}-T_{c}\right)^{2}}-\frac{1}{2} \dot{B}_{m} \frac{\left(t-T_{c}\right)^{4}}{\left(T_{m}-T_{c}\right)^{3}} \\
\dot{B}(t)=3 \dot{B}_{m} \frac{\left(t-T_{c}\right)^{2}}{\left(T_{m}-T_{c}\right)^{2}}-2 \dot{B}_{m} \frac{\left(t-T_{c}\right)^{3}}{\left(T_{m}-T_{c}\right)^{3}} \\
\ddot{B}(t)=6 \dot{B}_{m} \frac{\left(t-T_{c}\right)}{\left(T_{m}-T_{c}\right)^{2}}-6 \dot{B}_{m} \frac{\left(t-T_{c}\right)^{2}}{\left(T_{m}-T_{c}\right)^{3}}
\end{gathered}
$$

where $\dot{B}_{m}=87 \mathrm{G} / \mathrm{ms}$ is the maximum bdot. Thus

$$
\begin{gathered}
B\left(T_{c}\right)=B_{c}, \quad \dot{B}\left(T_{c}\right)=0, \quad \ddot{B}\left(T_{c}\right)=0 \\
B\left(T_{m}\right)=B_{c}+\frac{1}{2} \dot{B}_{m}\left(T_{m}-T_{c}\right)=1615.21 \text { Gauss } \\
\dot{B}\left(T_{m}\right)=\dot{B}_{m}, \quad \ddot{B}\left(T_{m}\right)=0 .
\end{gathered}
$$

For $T_{m} \leq t \leq T_{t}$ bdot is held fixed at $\dot{B}_{m}$. Thus we have

$$
B(t)=B\left(T_{m}\right)+\dot{B}_{m}\left(t-T_{m}\right), \quad \dot{B}(t)=\dot{B}_{m}, \quad \ddot{B}(t)=0
$$

and

$$
B\left(T_{t}\right)=B\left(T_{m}\right)+\dot{B}_{m}\left(T_{t}-T_{m}\right)=2063.26 \text { Gauss } .
$$

For $T_{t} \leq t \leq T_{p}$ we have

$$
\begin{gathered}
B(t)=B\left(T_{t}\right)+\dot{B}_{m}\left(t-T_{t}\right)-\dot{B}_{m} \frac{\left(t-T_{t}\right)^{3}}{\left(T_{p}-T_{t}\right)^{2}}+\frac{1}{2} \dot{B}_{m} \frac{\left(t-T_{t}\right)^{4}}{\left(T_{p}-T_{t}\right)^{3}} \\
\dot{B}(t)=\dot{B}_{m}-3 \dot{B}_{m} \frac{\left(t-T_{t}\right)^{2}}{\left(T_{p}-T_{t}\right)^{2}}+2 \dot{B}_{m} \frac{\left(t-T_{t}\right)^{3}}{\left(T_{p}-T_{t}\right)^{3}} \\
\ddot{B}(t)=-6 \dot{B}_{m} \frac{t-T_{t}}{\left(T_{p}-T_{t}\right)^{2}}+6 \dot{B}_{m} \frac{\left(t-T_{t}\right)^{2}}{\left(T_{p}-T_{t}\right)^{3}}
\end{gathered}
$$


Thus

$$
\begin{gathered}
B_{p}=B\left(T_{p}\right)=B\left(T_{t}\right)+\frac{1}{2} \dot{B}_{m}\left(T_{p}-T_{t}\right)=2554.81 \text { Gauss } \\
\dot{B}\left(T_{p}\right)=0, \quad \ddot{B}\left(T_{p}\right)=0 .
\end{gathered}
$$

The field on the merging porch is held fixed at $B_{p}$. Thus for $T_{p} \leq t \leq T_{f}$ we have

$$
B(t)=B_{p}, \quad \dot{B}(t)=0, \quad \ddot{B}(t)=0 .
$$

Figures 1 and 2 show $B(t)$ and $\dot{B}(t)$ for the time interval $0 \leq t \leq T_{p}$.

\subsection{Gap Volt Program for Capture and Acceleration}

We assume that the gap voltage $V(t)$ increases parabolically from zero to $V_{c}=0.5 \mathrm{kV}$ during capture time $T_{c}$. Thus for $0 \leq t \leq T_{c}$ we have

$$
V(t)=V_{c} t^{2} / T_{c}^{2}, \quad \dot{V}(t)=2 V_{c} t / T_{c}^{2}, \quad \ddot{V}(t)=2 V_{c} / T_{c}^{2}
$$

and therefore

$$
V\left(T_{c}\right)=V_{c}, \quad \dot{V}\left(T_{c}\right)=2 V_{c} / T_{c}=\dot{V}_{c}, \quad \ddot{V}\left(T_{c}\right)=2 V_{c} / T_{c}^{2} .
$$

For $T_{c} \leq t \leq T_{m}$ we have

$$
\begin{gathered}
V(t)=V_{c}+\dot{V}_{c}\left(t-T_{c}\right)+c\left(t-T_{c}\right)^{2}+d\left(t-T_{c}\right)^{3}+e\left(t-T_{c}\right)^{4} \\
\dot{V}(t)=\dot{V}_{c}+2 c\left(t-T_{c}\right)+3 d\left(t-T_{c}\right)^{2}+4 e\left(t-T_{c}\right)^{3} \\
\ddot{V}(t)=2 c+6 d\left(t-T_{c}\right)+12 e\left(t-T_{c}\right)^{2}
\end{gathered}
$$

where $\dot{V}_{c}$ is given by the second of equations (20). The parameters $c, d$, and $e$ are determined by the requirement that

$$
\ddot{V}\left(T_{c}\right)=\ddot{V}_{c}, \quad V\left(T_{m}\right)=V_{m}, \quad \dot{V}\left(T_{m}\right)=0 .
$$

Here $V_{m}=30 \mathrm{kV}$ and where $\ddot{V}_{c}$ is an adjustable parameter set to be 0.40 $\mathrm{G} / \mathrm{ms} / \mathrm{ms}$. Using (24) in (21-23) and solving for $c, d$, and $e$ we obtain

$$
2 c=\ddot{V}_{c}, \quad d=\frac{1}{T^{4}}\left\{4 D T-E T^{2}\right\}, \quad e=\frac{1}{T^{4}}\{-3 D+E T\}
$$

where $T=T_{m}-T_{c}$ and

$$
D=V_{m}-V_{c}-\dot{V}_{c} T-c T^{2}, \quad E=-\dot{V}_{c}-2 c T .
$$


Note that the functions $V(t)$ and $\dot{V}(t)$ defined by (21) and (22) and by (19) are continuous at time $T_{c}$. This is not true for $\ddot{V}(t)$ unless $\ddot{V}_{c}$ is given the value $2 V_{c} / T_{c}^{2}$. In general we need to give $\ddot{V}_{c}$ a different value in order to keep the bucket area and momentum spread from becoming too large after capture.

For $T_{m} \leq t \leq T_{t}$ the gap voltage is held constant at $V_{m}=30 \mathrm{kV}$. Thus

$$
V(t)=V_{m}, \quad \dot{V}(t)=0, \quad \ddot{V}(t)=0 .
$$

For $T_{t} \leq t \leq T_{p}$ we have

$$
\begin{gathered}
V(t)=V_{m}+4\left(V_{p}-V_{m}\right) \frac{\left(t-T_{t}\right)^{3}}{\left(T_{p}-T_{t}\right)^{3}}-3\left(V_{p}-V_{m}\right) \frac{\left(t-T_{t}\right)^{4}}{\left(T_{p}-T_{t}\right)^{4}} \\
\dot{V}(t)=12\left(V_{p}-V_{m}\right) \frac{\left(t-T_{t}\right)^{2}}{\left(T_{p}-T_{t}\right)^{3}}-12\left(V_{p}-V_{m}\right) \frac{\left(t-T_{t}\right)^{3}}{\left(T_{p}-T_{t}\right)^{4}} \\
\ddot{V}(t)=24\left(V_{p}-V_{m}\right) \frac{\left(t-T_{t}\right)}{\left(T_{p}-T_{t}\right)^{3}}-36\left(V_{p}-V_{m}\right) \frac{\left(t-T_{t}\right)^{2}}{\left(T_{p}-T_{t}\right)^{4}}
\end{gathered}
$$

where $V_{p}=1.0 \mathrm{kV}$ is the initial gap voltage on the merging porch.

The red curve in Figure 3 shows $V(t)$ for the time interval $0 \leq t \leq T_{p}$.

\subsection{Capture and Acceleration Parameters}

Capture and acceleration parameters are plotted in Figure 3. The blue and red curves show $\dot{B}(t) / 2$ and $V(t)$. The black and green curves are the synchronous phase and bucket area. As indicated by the legend, what is actually plotted is the bucket area times 10 . Here we see that the bucket area increases linearly during the $6 \mathrm{~ms}$ capture, oscillates slightly over the next $23 \mathrm{~ms}$, and then reaches a minimum of $2.1 \mathrm{eV}-\mathrm{s}$ as bdot reaches its maximum of 87 Gauss/ms. The violet curve shows the fractional momentum half-height, $\Delta p / p_{s}$, of the bucket. As indicated by the legend, what is actually plotted is $\Delta p / p_{s}$ times 10000 . The maximum $\Delta p / p_{s}=0.0042$ occurs at approximately $14 \mathrm{~ms}$. If the gap voltage

parameter $\ddot{V}_{c}$ is increased from its setting of 0.4 , then the maximum $\Delta p / p_{s}$ increases; if it is decreased, the bucket area takes too much of a dip just after capture. The setting of 0.4 is therefore a compromise between these two effects. Note that the maximum dispersion in Booster is $D_{x}=2.9$ meters, which, with $\Delta p / p_{s}=0.0042$, gives a closed orbit displacement of $\Delta x=\left(\Delta p / p_{s}\right) D_{x}=12 \mathrm{~mm}$. Keeping $\Delta p / p_{s}$ under control after capture is therefore very important. 


\subsection{Capture and Acceleration Simulation}

The simulation of capture and acceleration starts with an initial particle distribution and applies the turn-by-turn equations

$$
\begin{gathered}
\phi_{n+1}=\left\{\frac{\omega_{n+1}^{s}}{\omega_{n}^{s}}\right\} \phi_{n}+2 \pi h\left\{\frac{\omega_{n+1}^{s}}{\omega_{n}^{s}}\right\}\left(\frac{\omega_{n}^{s}-\omega_{n}}{\omega_{n}}\right) \\
W_{n+1}=\left\{\frac{\omega_{n}^{s}}{\omega_{n+1}^{s}}\right\} W_{n}+\left(\frac{2 \pi}{\omega_{n+1}^{s}}\right)\left\{F\left(\phi_{n+1}+\phi_{n+1}^{s}\right)-F\left(\phi_{n+1}^{s}\right)\right\}
\end{gathered}
$$

derived in the Appendix.

For the capture and acceleration of gold ions we have

$$
F(\phi)=A \sin \phi, \quad A=\left(\frac{e Q V}{2 \pi h}\right), \quad h=6
$$

where $V$ is the gap voltage. The initial particle distribution is that of completely unbunched beam. This is the situation in Booster just after the beam pulse from Tandem has been injected and before RF capture begins. As in Ref. [5], we assume a uniform distribution and consider a 50-by-50 rectangular array of points (particles) which cover the region occupied by one sixth of the beam. (This is the region of one RF bucket at harmonic $h=6$.) The array has boundaries at times $t= \pm \delta t$ and energies $e= \pm \delta e$. We shall take $\delta e=0.091 \mathrm{MeV}$. This corresponds to the momentum deviation $\delta p$ given by $\delta p / p_{s}=\delta e /\left(E_{s} \beta_{s}^{2}\right)=0.00025$ with $p_{s}, E_{s}$, and $\beta_{s}$ evaluated at injection. At harmonic $h=6$, the width of a single stationary bucket at injection is $2.518 \mu \mathrm{s}$, so we take $\delta t=1.259 \mu \mathrm{s}$. The longitudinal emittance of the array is $4(\delta t)(\delta e)=0.46 \mathrm{eV}-\mathrm{s}$.

Figure 4 shows the particle distribution in a single RF bucket at the end of the $6 \mathrm{~ms}$ capture period. Here the energy and time deviations are plotted for each particle and the rectangle outlines the region of the initial distribution. The final distribution shows a fair amount of filamentation of the initial emittance and we see that the tails of the distribution come close to the bucket separatrix. If the capture voltage $V_{c}$ is decreased from its setting of $0.5 \mathrm{kV}$, then some of the particles in the tails will be lost as the bucket area decreases just after capture. If $V_{c}$ is increased, there is greater filamentation of the emittance and this results in more loss as the bucket area reaches its minimum at maximum bdot. In this way $V_{c}=0.5$ was found to be the optimum setting for the $6 \mathrm{~ms}$ capture setup.

Figure 5 shows the particle distributions and RF buckets for two adjacent bunches at the end of acceleration onto the merging porch. Here the 
energy and time deviations (from the center of the bunch) for each plotted point are given by

$$
e=h \omega_{s} W, \quad t=\phi /\left(h \omega_{s}\right)
$$

where $h=3, \omega_{s}=2 \pi f_{s}$, and $f_{s}=0.270614 \mathrm{MHz}$ is the revolution frequency of the synchronous particle on the porch.

\subsection{Gap Volt Program for Merging}

In the merging scheme developed by J.M. Brennan, six gold bunches are merged into three. To accomplish this the harmonic 6 gap voltage is decreased linearly from its initial value $V_{p}$ to zero over merging time $T_{M}$. Concurrently the harmonic 3 voltage is increased linearly from zero to its final value $V_{f}$. Using $V_{6}$ and $V_{3}$ to denote the harmonic 6 and 3 voltages we have

$$
V_{6}(t)=\left(\frac{T_{f}-t}{T_{M}}\right) V_{p}, \quad V_{3}(t)=\left(\frac{t-T_{p}}{T_{M}}\right) V_{f}
$$

where

$$
T_{M}=T_{f}-T_{p}
$$

\subsection{Merge Simulation}

Starting with the two-bunch distribution shown in Figure $\mathbf{5}$ we apply the turn-by-turn equations derived in the Appendix. On the merging porch the field is constant, the synchronous phase is zero, and the turn-by-turn equations become

$$
\begin{gathered}
\phi_{n+1}=\phi_{n}+\left(\frac{2 \pi}{\omega_{s}}\right) a_{s} W_{n} \\
W_{n+1}=W_{n}+\left(\frac{2 \pi}{\omega_{s}}\right) F\left(\phi_{n+1}\right)
\end{gathered}
$$

where

$$
a_{s}=\left\{\frac{h^{2} \omega_{s}^{2} \eta_{s}}{\beta_{s}^{2} E_{s}}\right\}, \quad F(\phi)=A_{1} \sin \phi-A_{2} \sin 2 \phi
$$

and

$$
A_{1}=\left(\frac{e Q V_{3}}{2 \pi h}\right), \quad A_{2}=\left(\frac{e Q V_{6}}{2 \pi h}\right), \quad h=3 .
$$

Here $V_{3}$ and $V_{6}$ are given by (35) with $V_{p}=V_{f}=1.0 \mathrm{kV}$.

Figures 6, 7, 8, 9, 10, 11, and 12 show the evolution of the distribution as the bunches are merged. The violet curve in each figure shows the RF 
bucket separatrix. The total merging time here is $T_{M}=40 \mathrm{~ms}$ and the percentages in the figure captions refer to the fraction $\left(t-T_{p}\right) / T_{M}$ of merge completed. Figures 13 and 14 show the final distributions obtained with reduced merging times of 20 and $10 \mathrm{~ms}$ respectively.

\section{Deuteron Simulation}

\subsection{Magnetic Field Program}

As before, the field $B$ is defined during the time intervals

$$
0<T_{c}<T_{m}<T_{t}<T_{p}<T_{f}
$$

where $T_{c}$ is the capture time, $T_{m}$ is the time at which maximum bdot is reached, $T_{t}$ is the time at which the transition to zero bdot begins, $T_{p}$ is the time at which the merging porch begins, and $T_{f}$ is the final time at the end of the merging process. To obtain a magnetic cycle close to the one used in practice we take

$$
\begin{gathered}
T_{c}=6 \mathrm{~ms}, \quad T_{m}=T_{c}+23 \mathrm{~ms}=29 \mathrm{~ms} \\
T_{t}=T_{m}+17.44 \mathrm{~ms}=46.44 \mathrm{~ms}, \quad T_{p}=T_{t}+8.38 \mathrm{~ms}=54.82 \mathrm{~ms} .
\end{gathered}
$$

The duration $T_{f}-T_{p}$ of the merging process considered here will range from 10 to $40 \mathrm{~ms}$.

Capture takes place at constant field. Thus for $0 \leq t \leq T_{c}$ we have

$$
B(t)=B_{c}, \quad \dot{B}(t)=0, \quad \ddot{B}(t)=0
$$

where $B_{c}=615.97$ Gauss. (The dots denote differentiation with respect to time.)

For $T_{c} \leq t \leq T_{m}$ we have

$$
\begin{gathered}
B(t)=B_{c}+\dot{B}_{m} \frac{\left(t-T_{c}\right)^{3}}{\left(T_{m}-T_{c}\right)^{2}}-\frac{1}{2} \dot{B}_{m} \frac{\left(t-T_{c}\right)^{4}}{\left(T_{m}-T_{c}\right)^{3}} \\
\dot{B}(t)=3 \dot{B}_{m} \frac{\left(t-T_{c}\right)^{2}}{\left(T_{m}-T_{c}\right)^{2}}-2 \dot{B}_{m} \frac{\left(t-T_{c}\right)^{3}}{\left(T_{m}-T_{c}\right)^{3}} \\
\ddot{B}(t)=6 \dot{B}_{m} \frac{\left(t-T_{c}\right)}{\left(T_{m}-T_{c}\right)^{2}}-6 \dot{B}_{m} \frac{\left(t-T_{c}\right)^{2}}{\left(T_{m}-T_{c}\right)^{3}}
\end{gathered}
$$


where $\dot{B}_{m}=69 \mathrm{G} / \mathrm{ms}$ is the maximum bdot. Thus

$$
\begin{gathered}
B\left(T_{c}\right)=B_{c}, \quad \dot{B}\left(T_{c}\right)=0, \quad \ddot{B}\left(T_{c}\right)=0 \\
B\left(T_{m}\right)=B_{c}+\frac{1}{2} \dot{B}_{m}\left(T_{m}-T_{c}\right)=1409.47 \text { Gauss } \\
\dot{B}\left(T_{m}\right)=\dot{B}_{m}, \quad \ddot{B}\left(T_{m}\right)=0 .
\end{gathered}
$$

For $T_{m} \leq t \leq T_{t}$ bdot is held fixed at $\dot{B}_{m}$. Thus we have

$$
B(t)=B\left(T_{m}\right)+\dot{B}_{m}\left(t-T_{m}\right), \quad \dot{B}(t)=\dot{B}_{m}, \quad \ddot{B}(t)=0
$$

and

$$
B\left(T_{t}\right)=B\left(T_{m}\right)+\dot{B}_{m}\left(T_{t}-T_{m}\right)=2612.83 \text { Gauss }
$$

For $T_{t} \leq t \leq T_{p}$ we have

$$
\begin{gathered}
B(t)=B\left(T_{t}\right)+\dot{B}_{m}\left(t-T_{t}\right)-\dot{B}_{m} \frac{\left(t-T_{t}\right)^{3}}{\left(T_{p}-T_{t}\right)^{2}}+\frac{1}{2} \dot{B}_{m} \frac{\left(t-T_{t}\right)^{4}}{\left(T_{p}-T_{t}\right)^{3}} \\
\dot{B}(t)=\dot{B}_{m}-3 \dot{B}_{m} \frac{\left(t-T_{t}\right)^{2}}{\left(T_{p}-T_{t}\right)^{2}}+2 \dot{B}_{m} \frac{\left(t-T_{t}\right)^{3}}{\left(T_{p}-T_{t}\right)^{3}} \\
\ddot{B}(t)=-6 \dot{B}_{m} \frac{t-T_{t}}{\left(T_{p}-T_{t}\right)^{2}}+6 \dot{B}_{m} \frac{\left(t-T_{t}\right)^{2}}{\left(T_{p}-T_{t}\right)^{3}} .
\end{gathered}
$$

Thus

$$
\begin{gathered}
B_{p}=B\left(T_{p}\right)=B\left(T_{t}\right)+\frac{1}{2} \dot{B}_{m}\left(T_{p}-T_{t}\right)=2901.94 \text { Gauss } \\
\dot{B}\left(T_{p}\right)=0, \quad \ddot{B}\left(T_{p}\right)=0 .
\end{gathered}
$$

The field on the merging porch is held fixed at $B_{p}$. Thus for $T_{p} \leq t \leq T_{f}$ we have

$$
B(t)=B_{p}, \quad \dot{B}(t)=0, \quad \ddot{B}(t)=0 .
$$

Figures 15 and 16 show $B(t)$ and $\dot{B}(t)$ for the time interval $0 \leq t \leq T_{p}$. 


\subsection{Gap Volt Program for Capture and Acceleration}

As before, we assume that the gap voltage $V(t)$ increases parabolically from zero to $V_{c}=0.5 \mathrm{kV}$ during capture time $T_{c}$. Thus for $0 \leq t \leq T_{c}$ we have

$$
V(t)=V_{c} t^{2} / T_{c}^{2}, \quad \dot{V}(t)=2 V_{c} t / T_{c}^{2}, \quad \ddot{V}(t)=2 V_{c} / T_{c}^{2}
$$

and therefore

$$
V\left(T_{c}\right)=V_{c}, \quad \dot{V}\left(T_{c}\right)=2 V_{c} / T_{c}=\dot{V}_{c}, \quad \ddot{V}\left(T_{c}\right)=2 V_{c} / T_{c}^{2} .
$$

For $T_{c} \leq t \leq T_{m}$ we have

$$
\begin{gathered}
V(t)=V_{c}+\dot{V}_{c}\left(t-T_{c}\right)+c\left(t-T_{c}\right)^{2}+d\left(t-T_{c}\right)^{3}+e\left(t-T_{c}\right)^{4} \\
\dot{V}(t)=\dot{V}_{c}+2 c\left(t-T_{c}\right)+3 d\left(t-T_{c}\right)^{2}+4 e\left(t-T_{c}\right)^{3} \\
\ddot{V}(t)=2 c+6 d\left(t-T_{c}\right)+12 e\left(t-T_{c}\right)^{2}
\end{gathered}
$$

where $\dot{V}_{c}$ is given by the second of equations (60). The parameters $c, d$, and $e$ are determined by the requirement that

$$
\ddot{V}\left(T_{c}\right)=\ddot{V}_{c}, \quad V\left(T_{m}\right)=V_{m}, \quad \dot{V}\left(T_{m}\right)=0 .
$$

Here $V_{m}=25 \mathrm{kV}$ and where $\ddot{V}_{c}$ is an adjustable parameter set to be 0.35 $\mathrm{G} / \mathrm{ms} / \mathrm{ms}$. Using (64) in (61-63) and solving for $c, d$, and $e$ we obtain

$$
2 c=\ddot{V}_{c}, \quad d=\frac{1}{T^{4}}\left\{4 D T-E T^{2}\right\}, \quad e=\frac{1}{T^{4}}\{-3 D+E T\}
$$

where $T=T_{m}-T_{c}$ and

$$
D=V_{m}-V_{c}-\dot{V}_{c} T-c T^{2}, \quad E=-\dot{V}_{c}-2 c T .
$$

Note that the functions $V(t)$ and $\dot{V}(t)$ defined by (61) and (62) and by (59) are continuous at time $T_{c}$. This is not true for $\ddot{V}(t)$ unless $\ddot{V}_{c}$ is given the value $2 V_{c} / T_{c}^{2}$. In general we need to give $\ddot{V}_{c}$ a different value in order to keep the bucket area and momentum spread from becoming too large after capture.

For $T_{m} \leq t \leq T_{t}$ the gap voltage is held constant at $V_{m}=25 \mathrm{kV}$. Thus

$$
V(t)=V_{m}, \quad \dot{V}(t)=0, \quad \ddot{V}(t)=0 .
$$


For $T_{t} \leq t \leq T_{p}$ we have

$$
\begin{gathered}
V(t)=V_{m}+4\left(V_{p}-V_{m}\right) \frac{\left(t-T_{t}\right)^{3}}{\left(T_{p}-T_{t}\right)^{3}}-3\left(V_{p}-V_{m}\right) \frac{\left(t-T_{t}\right)^{4}}{\left(T_{p}-T_{t}\right)^{4}} \\
\dot{V}(t)=12\left(V_{p}-V_{m}\right) \frac{\left(t-T_{t}\right)^{2}}{\left(T_{p}-T_{t}\right)^{3}}-12\left(V_{p}-V_{m}\right) \frac{\left(t-T_{t}\right)^{3}}{\left(T_{p}-T_{t}\right)^{4}} \\
\ddot{V}(t)=24\left(V_{p}-V_{m}\right) \frac{\left(t-T_{t}\right)}{\left(T_{p}-T_{t}\right)^{3}}-36\left(V_{p}-V_{m}\right) \frac{\left(t-T_{t}\right)^{2}}{\left(T_{p}-T_{t}\right)^{4}}
\end{gathered}
$$

where $V_{p}=1.0 \mathrm{kV}$ is the initial gap voltage on the merging porch.

The red curve in Figure 17 shows $V(t)$ for the time interval $0 \leq t \leq T_{p}$.

\subsection{Capture and Acceleration Parameters}

Capture and acceleration parameters are plotted in Figure 17. The blue and red curves show $\dot{B}(t) / 2$ and $V(t)$. The black and green curves are the synchronous phase and bucket area. As indicated by the legend, what is actually plotted is the bucket area times 100 . Here we see that the bucket area increases linearly during the $6 \mathrm{~ms}$ capture, oscillates slightly over the next $23 \mathrm{~ms}$, and then reaches a minimum of $0.23 \mathrm{eV}-\mathrm{s}$ as bdot reaches its maximum of 69 Gauss/ms. The violet curve shows the fractional momentum half-height, $\Delta p / p_{s}$, of the bucket. The maximum $\Delta p / p_{s}=0.0045$ occurs at approximately $15 \mathrm{~ms}$.

\subsection{Capture and Acceleration Simulation}

The simulation of capture and acceleration starts with an initial particle distribution and applies the turn-by-turn equations

$$
\begin{gathered}
\phi_{n+1}=\left\{\frac{\omega_{n+1}^{s}}{\omega_{n}^{s}}\right\} \phi_{n}+2 \pi h\left\{\frac{\omega_{n+1}^{s}}{\omega_{n}^{s}}\right\}\left(\frac{\omega_{n}^{s}-\omega_{n}}{\omega_{n}}\right) \\
W_{n+1}=\left\{\frac{\omega_{n}^{s}}{\omega_{n+1}^{s}}\right\} W_{n}+\left(\frac{2 \pi}{\omega_{n+1}^{s}}\right)\left\{F\left(\phi_{n+1}+\phi_{n+1}^{s}\right)-F\left(\phi_{n+1}^{s}\right)\right\}
\end{gathered}
$$

derived in the Appendix.

For the capture and acceleration of Deuterons we have

$$
F(\phi)=A \sin \phi, \quad A=\left(\frac{e Q V}{2 \pi h}\right), \quad h=2
$$


where $V$ is the gap voltage. The initial particle distribution is that of completely unbunched beam. This is the situation in Booster just after the beam pulse from Tandem has been injected and before RF capture begins. We assume a uniform distribution and consider a 50-by-50 rectangular array of points (particles) which cover the region occupied by half of the beam. (This is the region of one RF bucket at harmonic $h=2$.) The array has boundaries at times $t= \pm \delta t$ and energies $e= \pm \delta e$. We shall take $\delta e=0.0086 \mathrm{MeV}$. This corresponds to the momentum deviation $\delta p$ given by $\delta p / p_{s}=\delta e /\left(E_{s} \beta_{s}^{2}\right)=0.00025$ with $p_{s}, E_{s}$, and $\beta_{s}$ evaluated at injection. At harmonic $h=2$, the width of a single stationary bucket at injection is $2.488 \mu \mathrm{s}$, so we take $\delta t=1.244 \mu \mathrm{s}$. The longitudinal emittance of the array is $4(\delta t)(\delta e)=0.0428 \mathrm{eV}$-s.

Figure 18 shows the particle distribution in a single RF bucket at the end of the $6 \mathrm{~ms}$ capture period. Here the energy and time deviations are plotted for each particle and the rectangle outlines the region of the initial distribution. The final distribution shows a fair amount of filamentation of the initial emittance and we see that the tails of the distribution come close to the bucket separatrix. If the capture voltage $V_{c}$ is decreased from its setting of $0.5 \mathrm{kV}$, then some of the particles in the tails will be lost as the bucket area decreases just after capture. If $V_{c}$ is increased, there is greater filamentation of the emittance and this results in more loss as the bucket area reaches its minimum at maximum bdot.

Figure 19 shows the particle distributions and RF buckets for two adjacent bunches at the end of acceleration onto the merging porch. Here the energy and time deviations (from the center of the bunch) for each plotted point are given by

$$
e=h \omega_{s} W, \quad t=\phi /\left(h \omega_{s}\right)
$$

where $h=1, \omega_{s}=2 \pi f_{s}$, and $f_{s}=0.803675 \mathrm{MHz}$ is the revolution frequency of the synchronous particle on the porch.

\subsection{Gap Volt Program for Merging}

In the merging scheme developed by J.M. Brennan, two deuteron bunches are merged into one. To accomplish this the harmonic 2 gap voltage is decreased linearly from its initial value $V_{p}$ to zero over merging time $T_{M}$. Concurrently the harmonic 1 voltage is increased linearly from zero to its final value $V_{f}$. Using $V_{2}$ and $V_{1}$ to denote the harmonic 2 and 1 voltages 
we have

$$
V_{2}(t)=\left(\frac{T_{f}-t}{T_{M}}\right) V_{p}, \quad V_{1}(t)=\left(\frac{t-T_{p}}{T_{M}}\right) V_{f}
$$

where

$$
T_{M}=T_{f}-T_{p}
$$

\subsection{Merge Simulation}

Starting with the two-bunch distribution shown in Figure 19 we apply the turn-by-turn equations derived in the Appendix. On the merging porch the field is constant, the synchronous phase is zero, and the turn-by-turn equations become

$$
\begin{gathered}
\phi_{n+1}=\phi_{n}+\left(\frac{2 \pi}{\omega_{s}}\right) a_{s} W_{n} \\
W_{n+1}=W_{n}+\left(\frac{2 \pi}{\omega_{s}}\right) F\left(\phi_{n+1}\right)
\end{gathered}
$$

where

$$
a_{s}=\left\{\frac{h^{2} \omega_{s}^{2} \eta_{s}}{\beta_{s}^{2} E_{s}}\right\}, \quad F(\phi)=A_{1} \sin \phi-A_{2} \sin 2 \phi
$$

and

$$
A_{1}=\left(\frac{e Q V_{1}}{2 \pi h}\right), \quad A_{2}=\left(\frac{e Q V_{2}}{2 \pi h}\right), \quad h=1 .
$$

Here $V_{1}$ and $V_{2}$ are given by (75) with $V_{p}=V_{f}=1.0 \mathrm{kV}$.

Figure 20 shows the final distribution obtained with a merging time $T_{M}=40 \mathrm{~ms}$. The violet curve in the figure shows the RF bucket separatrix. Figures 21 and $\mathbf{2 2}$ show the final distributions obtained with reduced merging times of 20 and $10 \mathrm{~ms}$ respectively.

\section{Appendix: Turn-by-Turn Equations for Longitudinal Motion}

Turn-by-turn equations for the longitudinal motion are derived here. The treatment is similar to that of MacLachlan $[6,7]$. We consider a ring with a single RF gap. The radius and circumference of the design orbit are $R$ and $2 \pi R$ respectively. The radius of curvature along the design orbit is $\rho$. The transition gamma is $\gamma_{t}$. The effect of the electric field generated by the ramping magnetic field is ignored and assumed to be negligible. 


\subsection{Synchronous Parameters}

Let $b_{n}^{s}$ and $R_{n}^{s}$ be the field and radius for the synchronous particle on its $n$th turn around the machine. We assume that these are given and calculate the other parameters of the synchronous particle in terms of these. Thus the radius of curvature is

$$
\rho_{n}^{s}=\rho\left(R_{n}^{s} / R\right)^{1 / \alpha}, \quad \alpha=\frac{1}{\gamma_{t}^{2}}
$$

and the momentum and energy just after the $n$th pass through the RF gap are

$$
c p_{n}^{s}=e Q b_{n}^{s} \rho_{n}^{s}, \quad E_{n}^{s}=\sqrt{\left(c p_{n}^{s}\right)^{2}+m^{2} c^{4}} .
$$

Here e is the proton charge, and $e Q$ and $m$ are the charge and mass of the particle. The synchronous beta, gamma, and angular frequency are

$$
\beta_{n}^{s}=c p_{n}^{s} / E_{n}^{s}, \quad \gamma_{n}^{s}=E_{n}^{s} /\left(m c^{2}\right), \quad \omega_{n}^{s}=c \beta_{n}^{s} / R_{n}^{s} .
$$

We also define the phase slip factor

$$
\eta_{n}^{s}=\alpha-\left(\frac{1}{\gamma_{n}^{s}}\right)^{2}=\frac{1}{\gamma_{t}^{2}}-\left(\frac{1}{\gamma_{n}^{s}}\right)^{2} .
$$

\subsection{Time Equation}

Let $T_{n}^{s}$ and $T_{n}$ be respectively the times at which the synchronous and asynchronous particles make their $n$th pass through the gap. Then

$$
T_{n+1}^{s}=T_{n}^{s}+2 \pi / \omega_{n}^{s}, \quad T_{n+1}=T_{n}+2 \pi / \omega_{n}
$$

where $\omega_{n}$ is the angular frequency of the asynchronous particle just after its $n$th pass through the gap. Defining

$$
t_{n}=T_{n}-T_{n}^{s}, \quad t_{n+1}=T_{n+1}-T_{n+1}^{s}
$$

we then have

$$
t_{n+1}=t_{n}+2 \pi\left(\frac{1}{\omega_{n}}-\frac{1}{\omega_{n}^{s}}\right)=t_{n}+\frac{2 \pi}{\omega_{n}^{s}}\left(\frac{\omega_{n}^{s}-\omega_{n}}{\omega_{n}}\right) .
$$




\subsection{Energy Equation}

Let $E_{n}$ be the energy of the asynchronous particle just after its $n$th pass through the gap. The momentum, beta, radius of curvature, radius, and angular frequency of the particle are then

$$
c p_{n}=\sqrt{E_{n}^{2}-m^{2} c^{4}}, \quad \beta_{n}=c p_{n} / E_{n}
$$

and

$$
\rho_{n}=\frac{c p_{n}}{e Q b_{n}}, \quad R_{n}=R\left(\rho_{n} / \rho\right)^{\alpha}, \quad \omega_{n}=c \beta_{n} / R_{n} .
$$

We shall assume that $b_{n}=b_{n}^{s}$.

Just after the next pass through the gap we have

$$
E_{n+1}=E_{n}+e Q V\left(T_{n+1}\right)
$$

where $V\left(T_{n+1}\right)$ is the voltage across the gap at time

$$
T_{n+1}=T_{n+1}^{s}+t_{n+1} .
$$

At the synchronous times $T_{n+1}^{s}$ the gap voltage must satisfy

$$
e Q V\left(T_{n+1}^{s}\right)=E_{n+1}^{s}-E_{n}^{s} .
$$

Defining

$$
e_{n}=E_{n}-E_{n}^{s}, \quad e_{n+1}=E_{n+1}-E_{n+1}^{s}
$$

we then have

$$
e_{n+1}=e_{n}+e Q\left\{V\left(T_{n+1}^{s}+t_{n+1}\right)-V\left(T_{n+1}^{s}\right)\right\}
$$

which together with

$$
t_{n+1}=t_{n}+\frac{2 \pi}{\omega_{n}^{s}}\left(\frac{\omega_{n}^{s}-\omega_{n}}{\omega_{n}}\right)
$$

gives the turn-by-turn longitudinal motion of the particle.

\subsection{Symplectic Map}

Since the longitudinal emittance is conserved, we want the map from $\left(t_{n}\right.$, $\left.e_{n}\right)$ to $\left(t_{n+1}, e_{n+1}\right)$ to be symplectic. The Jacobian matrix elements of the map are

$$
\frac{\partial t_{n+1}}{\partial t_{n}}=1, \quad \frac{\partial t_{n+1}}{\partial e_{n}}=2 \pi \frac{\partial\left(1 / \omega_{n}\right)}{\partial e_{n}}
$$




$$
\frac{\partial e_{n+1}}{\partial t_{n}}=e Q V^{\prime}, \quad \frac{\partial e_{n+1}}{\partial e_{n}}=1+2 \pi e Q V^{\prime} \frac{\partial\left(1 / \omega_{n}\right)}{\partial e_{n}}
$$

where $V^{\prime}$ is the derivative of $V$ with respect to $T$ at time $T_{n+1}$. Thus we have

$$
\left(\frac{\partial t_{n+1}}{\partial t_{n}}\right)\left(\frac{\partial e_{n+1}}{\partial e_{n}}\right)-\left(\frac{\partial t_{n+1}}{\partial e_{n}}\right)\left(\frac{\partial e_{n+1}}{\partial t_{n}}\right)=1
$$

which shows that the map is symplectic. If $t_{n}=0$ and $e_{n}=0$, then it follows from (94) and (95) that $t_{n+1}=0$ and $e_{n+1}=0$. Thus if $t_{1}=0$ and $e_{1}=0$ it follows by induction that $t_{n}=0$ and $e_{n}=0$ for all $n$, and we see that the point $\left(t_{1}, e_{1}\right)=(0,0)$ is a fixed point.

\subsection{Approximate Time Equation}

Now

$$
\frac{\omega_{n}^{s}}{\omega_{n}}=1-\left(\frac{\omega_{n}-\omega_{n}^{s}}{\omega_{n}^{s}}\right)+\left(\frac{\omega_{n}-\omega_{n}^{s}}{\omega_{n}^{s}}\right)^{2}-\left(\frac{\omega_{n}-\omega_{n}^{s}}{\omega_{n}^{s}}\right)^{3}+\cdots
$$

and, to first order in $p_{n}-p_{n}^{s}$ and $E_{n}-E_{n}^{s}$,

$$
\left(\frac{\omega_{n}-\omega_{n}^{s}}{\omega_{n}^{s}}\right)=-\eta_{n}^{s}\left(\frac{p_{n}-p_{n}^{s}}{p_{n}^{s}}\right)=-\eta_{n}^{s}\left\{\frac{E_{n}-E_{n}^{s}}{\left(\beta_{n}^{s}\right)^{2} E_{n}^{s}}\right\}
$$

Thus, to first order we have

$$
\frac{\omega_{n}^{s}}{\omega_{n}}=1-\left(\frac{\omega_{n}-\omega_{n}^{s}}{\omega_{n}^{s}}\right)=1+\eta_{s}\left\{\frac{E_{n}-E_{n}^{s}}{\left(\beta_{n}^{s}\right)^{2} E_{n}^{s}}\right\}=1+\left\{\frac{\eta_{n}^{s}}{\left(\beta_{n}^{s}\right)^{2} E_{n}^{s}}\right\} e_{n}
$$

and equation (95) becomes

$$
t_{n+1}=t_{n}+\frac{2 \pi}{\omega_{n}^{s}}\left\{\frac{\eta_{n}^{s}}{\left(\beta_{n}^{s}\right)^{2} E_{n}^{s}}\right\} e_{n} .
$$

This, together with

$$
e_{n+1}=e_{n}+e Q\left\{V\left(T_{n+1}^{s}+t_{n+1}\right)-V\left(T_{n+1}^{s}\right)\right\}
$$

again produces a symplectic map from $\left(t_{n}, e_{n}\right)$ to $\left(t_{n+1}, e_{n+1}\right)$. 


\subsection{Gap Voltage}

We assume that the gap voltage is of the form

$$
V(T)=\left(\frac{2 \pi h}{e Q}\right) F\{\psi(T)\}
$$

where

$$
F(\psi+2 \pi)=F(\psi)
$$

and

$$
\psi\left(T_{n}^{s}\right)=2 \pi h n+\phi_{n}^{s} .
$$

We call $\phi_{n}^{s}$ the synchronous phase for the $n$th turn. Putting (106) in (104) and using (105) we have

$$
e Q V\left(T_{n}^{s}\right)=2 \pi h F\left(\phi_{n}^{s}\right)
$$

and since

$$
e Q V\left(T_{n+1}^{s}\right)=E_{n+1}^{s}-E_{n}^{s}
$$

we have

$$
2 \pi h F\left(\phi_{n+1}^{s}\right)=E_{n+1}^{s}-E_{n}^{s} .
$$

This equation determines the synchronous phases $\phi_{n}^{s}$.

For times $T$ such that

$$
T_{m}^{s}<T<T_{m+1}^{s}
$$

we assume that

$$
\psi(T)=\psi\left(T_{m}^{s}\right)+\left(T-T_{m}^{s}\right) h \omega_{m}^{s}\left(1+\epsilon_{m}^{s}\right)
$$

where

$$
\epsilon_{m}^{s}=\left(\phi_{m+1}^{s}-\phi_{m}^{s}\right) /(2 \pi h) .
$$

Thus for

$$
T_{m}^{s}<T_{n}^{s}+t_{n}<T_{m+1}^{s}
$$

we have

$$
\psi\left(T_{n}^{s}+t_{n}\right)=\psi\left(T_{m}^{s}\right)+\left(T_{n}^{s}+t_{n}-T_{m}^{s}\right) h \omega_{m}^{s}\left(1+\epsilon_{m}^{s}\right) .
$$




\subsection{Phase and $W$ Equations}

Defining

$$
\phi_{n}=\psi\left(T_{n}\right)-\psi\left(T_{n}^{s}\right)=\psi\left(T_{n}^{s}+t_{n}\right)-\psi\left(T_{n}^{s}\right)
$$

and using (114), we have

$$
\phi_{n}=t_{n} h \omega_{n}^{s}+\zeta_{1}+\zeta_{2}+\zeta_{3}
$$

where

$$
\begin{gathered}
\zeta_{1}=2 \pi h(m-n)+\left(T_{n}^{s}-T_{m}^{s}\right) h \omega_{m}^{s}\left(1+\epsilon_{m}^{s}\right), \\
\zeta_{2}=t_{n}\left\{h \omega_{m}^{s}\left(1+\epsilon_{m}^{s}\right)-h \omega_{n}^{s}\right\}, \quad \zeta_{3}=\phi_{m}^{s}-\phi_{n}^{s} .
\end{gathered}
$$

The terms $\zeta_{i}$ are small (or vanish) and to a good approximation we can write

$$
\phi_{n}=t_{n} h \omega_{n}^{s}, \quad \phi_{n+1}=t_{n+1} h \omega_{n+1}^{s}
$$

where

$$
t_{n+1}=t_{n}+\frac{2 \pi}{\omega_{n}^{s}}\left(\frac{\omega_{n}^{s}-\omega_{n}}{\omega_{n}}\right)
$$

Thus we have

$$
\frac{\phi_{n+1}}{h \omega_{n+1}^{s}}=\frac{\phi_{n}}{h \omega_{n}^{s}}+\frac{2 \pi}{\omega_{n}^{s}}\left(\frac{\omega_{n}^{s}-\omega_{n}}{\omega_{n}}\right)
$$

and therefore

$$
\phi_{n+1}=\left\{\frac{\omega_{n+1}^{s}}{\omega_{n}^{s}}\right\} \phi_{n}+2 \pi h\left\{\frac{\omega_{n+1}^{s}}{\omega_{n}^{s}}\right\}\left(\frac{\omega_{n}^{s}-\omega_{n}}{\omega_{n}}\right) .
$$

Let us now define

$$
W_{n}=e_{n} /\left(h \omega_{n}^{s}\right)
$$

where

$$
e_{n+1}=e_{n}+e Q\left\{V\left(T_{n+1}^{s}+t_{n+1}\right)-V\left(T_{n+1}^{s}\right)\right\} .
$$

Then we have

$$
W_{n+1}=\left\{\frac{\omega_{n}^{s}}{\omega_{n+1}^{s}}\right\} W_{n}+\left(\frac{e Q}{h \omega_{n+1}^{s}}\right)\left\{V\left(T_{n+1}^{s}+t_{n+1}\right)-V\left(T_{n+1}^{s}\right)\right\} .
$$

Now since

$$
\psi\left(T_{n+1}^{s}+t_{n+1}\right)=\psi\left(T_{n+1}\right)=\phi_{n+1}+\psi\left(T_{n+1}^{s}\right)
$$

and

$$
\psi\left(T_{n+1}^{s}\right)=2 \pi h(n+1)+\phi_{n+1}^{s}
$$


we have

$$
\psi\left(T_{n+1}^{s}+t_{n+1}\right)=\phi_{n+1}+\phi_{n+1}^{s}+2 \pi h(n+1) .
$$

Thus

$$
\begin{gathered}
V\left(T_{n+1}^{s}+t_{n+1}\right)=\left(\frac{2 \pi h}{e Q}\right) F\left(\phi_{n+1}+\phi_{n+1}^{s}\right) \\
V\left(T_{n+1}^{s}\right)=\left(\frac{2 \pi h}{e Q}\right) F\left(\phi_{n+1}^{s}\right)
\end{gathered}
$$

and equation (125) becomes

$$
W_{n+1}=\left\{\frac{\omega_{n}^{s}}{\omega_{n+1}^{s}}\right\} W_{n}+\left(\frac{2 \pi}{\omega_{n+1}^{s}}\right)\left\{F\left(\phi_{n+1}+\phi_{n+1}^{s}\right)-F\left(\phi_{n+1}^{s}\right)\right\} .
$$

This together with (122) gives the longitudinal motion of the particle in terms of $\phi_{n}$ and $W_{n}$. Writing these equations as

$$
\begin{gathered}
\phi_{n+1}=A \phi_{n}+2 \pi h A\left(\frac{\omega_{n}^{s}-\omega_{n}}{\omega_{n}}\right) \\
W_{n+1}=\frac{1}{A} W_{n}+\frac{1}{A}\left(\frac{2 \pi}{\omega_{n}^{s}}\right)\left\{F\left(\phi_{n+1}+\phi_{n+1}^{s}\right)-F\left(\phi_{n+1}^{s}\right)\right\}
\end{gathered}
$$

where

$$
A=\frac{\omega_{n+1}^{s}}{\omega_{n}^{s}}
$$

we have

$$
\begin{gathered}
\frac{\partial \phi_{n+1}}{\partial \phi_{n}}=A, \quad \frac{\partial \phi_{n+1}}{\partial W_{n}}=2 \pi h A \omega_{n}^{s} \frac{\partial\left(1 / \omega_{n}\right)}{\partial W_{n}} \\
\frac{\partial W_{n+1}}{\partial \phi_{n}}=\frac{2 \pi}{\omega_{n}^{s}} F^{\prime}, \quad \frac{\partial W_{n+1}}{\partial W_{n}}=\frac{1}{A}+4 \pi^{2} h F^{\prime} \frac{\partial\left(1 / \omega_{n}\right)}{\partial W_{n}} .
\end{gathered}
$$

Here $F^{\prime}$ is the derivative of $F$ with respect to $\phi$ at phase $\phi_{n+1}+\phi_{n+1}^{s}$. Thus

$$
\left(\frac{\partial \phi_{n+1}}{\partial \phi_{n}}\right)\left(\frac{\partial W_{n+1}}{\partial W_{n}}\right)-\left(\frac{\partial \phi_{n+1}}{\partial W_{n}}\right)\left(\frac{\partial W_{n+1}}{\partial \phi_{n}}\right)=1
$$

which shows that the map from $\left(\phi_{n}, W_{n}\right)$ to $\left(\phi_{n+1}, W_{n+1}\right)$ is symplectic. 


\subsection{Hamiltonian Equations of Motion}

The map given by (132) and (133) is symplectic for any $A \neq 0$ and in particular for $A=1$. In this case we have

$$
\begin{gathered}
\phi_{n+1}=\phi_{n}+2 \pi h\left(\frac{\omega_{n}^{s}-\omega_{n}}{\omega_{n}}\right) \\
W_{n+1}=W_{n}+\left(\frac{2 \pi}{\omega_{n}^{s}}\right)\left\{F\left(\phi_{n+1}+\phi_{n+1}^{s}\right)-F\left(\phi_{n+1}^{s}\right)\right\} .
\end{gathered}
$$

Since the difference between $\omega_{n+1}^{s}$ and $\omega_{n}^{s}$ is very small, these equations are a good approximation to equations (132-134). Using the further approximation

$$
\left(\frac{\omega_{n}^{s}-\omega_{n}}{\omega_{n}}\right)=\left\{\frac{\eta_{n}^{s}}{\left(\beta_{n}^{s}\right)^{2} E_{n}^{s}}\right\} e_{n}=\left\{\frac{h \omega_{n}^{s} \eta_{n}^{s}}{\left(\beta_{n}^{s}\right)^{2} E_{n}^{s}}\right\} W_{n}
$$

we have

$$
\begin{gathered}
\phi_{n+1}=\phi_{n}+\left(\frac{2 \pi}{\omega_{n}^{s}}\right) a_{n}^{s} W_{n} \\
W_{n+1}=W_{n}+\left(\frac{2 \pi}{\omega_{n}^{s}}\right)\left\{F\left(\phi_{n+1}+\phi_{n+1}^{s}\right)-F\left(\phi_{n+1}^{s}\right)\right\}
\end{gathered}
$$

where

$$
a_{n}^{s}=\left\{\frac{h^{2}\left(\omega_{n}^{s}\right)^{2} \eta_{n}^{s}}{\left(\beta_{n}^{s}\right)^{2} E_{n}^{s}}\right\}=\left\{\frac{h^{2} c^{2} \eta_{n}^{s}}{\left(R_{n}^{s}\right)^{2} E_{n}^{s}}\right\} .
$$

This again produces a symplectic map.

The turn-by-turn motion given by (141) and (142) is approximated by the motion that follows from the Hamiltonian

$$
H(\phi, W)=\frac{1}{2} a W^{2}+U(\phi)
$$

where

$$
a=\left\{\frac{h^{2} \omega_{s}^{2} \eta_{s}}{\beta_{s}^{2} E_{s}}\right\}=\left\{\frac{h^{2} c^{2} \eta_{s}}{R_{s}^{2} E_{s}}\right\}, \quad \frac{\partial U}{\partial \phi}=-F\left(\phi+\phi_{s}\right)+F\left(\phi_{s}\right) .
$$

Here the subscript $s$ denotes the parameters of the synchronous particle. The equations of motion are

$$
\dot{\phi}=\frac{d \phi}{d t}=\frac{\partial H}{\partial W}=a W
$$




$$
\dot{W}=\frac{d W}{d t}=-\frac{\partial H}{\partial \phi}=-\frac{\partial U}{\partial \phi}=F\left(\phi+\phi_{s}\right)-F\left(\phi_{s}\right) .
$$

First-order symplectic integration [8] of these over time $T_{s}=2 \pi / \omega_{s}$ yields equations (141) and (142).

We note here that for the case in which

$$
F(\psi)=A_{1} \sin \psi-A_{2} \sin 2 \psi
$$

we have

$$
\begin{gathered}
\frac{\partial U}{\partial \phi}=-A_{1} \sin \left(\phi+\phi_{s}\right)+A_{2} \sin \left(2 \phi+2 \phi_{s}\right)+A_{1} \sin \phi_{s}-A_{2} \sin 2 \phi_{s} \\
\frac{\partial^{2} U}{\partial^{2} \phi}=-A_{1} \cos \left(\phi+\phi_{s}\right)+2 A_{2} \cos \left(2 \phi+2 \phi_{s}\right)
\end{gathered}
$$

and

$$
U(\phi)=A_{1} \cos \left(\phi+\phi_{s}\right)-\frac{1}{2} A_{2} \cos \left(2 \phi+2 \phi_{s}\right)+C \phi+D
$$

where

$$
C=A_{1} \sin \phi_{s}-A_{2} \sin 2 \phi_{s}, \quad D=-A_{1} \cos \phi_{s}+\frac{1}{2} A_{2} \cos 2 \phi_{s} .
$$

\subsection{Stable and Unstable Fixed Points}

The fixed points $\left(\phi_{f}, W_{f}\right)$ of the motion generated by the Hamiltonian satisfy the equations

$$
0=\frac{\partial H}{\partial \phi}=\frac{\partial U}{\partial \phi}=-F\left(\phi+\phi_{s}\right)+F\left(\phi_{s}\right), \quad 0=\frac{\partial H}{\partial W}=a W .
$$

Thus

$$
F\left(\phi_{f}+\phi_{s}\right)=F\left(\phi_{s}\right), \quad W_{f}=0 .
$$

To determine whether the motion near a fixed point is stable or unstable we must examine the second derivative of $U$ with respect to $\phi$. Let $U_{\phi \phi}$ be the value of the second derivative at the fixed point. Then, when $a<0$ (below transition), the motion near the fixed point will be stable if $U_{\phi \phi}<0$ and unstable if $U_{\phi \phi}>0$. Similarly when $a>0$ (above transition), the motion near the fixed point will be stable if $U_{\phi \phi}>0$ and unstable if $U_{\phi \phi}<0$.

For the case in which

$$
F(\psi)=A \sin \psi
$$


the fixed points are given by

$$
\sin \left(\phi_{f}+\phi_{s}\right)=\sin \phi_{s}
$$

and we have

$$
U_{\phi \phi}=-A \cos \left(\phi_{f}+\phi_{s}\right) .
$$

If we are below transition with $A>0$ and $0 \leq \phi_{s}<\pi / 2$, then the motion near fixed point $\phi_{f}=0$ will be stable and the motion near $\phi_{f}=\pi-2 \phi_{s}$ will be unstable.

For the case in which

$$
F(\psi)=A_{1} \sin \psi-A_{2} \sin 2 \psi
$$

the fixed points are given by

$$
A_{1} \sin \left(\phi_{f}+\phi_{s}\right)-A_{2} \sin \left(2 \phi_{f}+2 \phi_{s}\right)=A_{1} \sin \phi_{s}-A_{2} \sin 2 \phi_{s}
$$

and we have

$$
U_{\phi \phi}=-A_{1} \cos \left(\phi_{f}+\phi_{s}\right)+2 A_{2} \cos \left(2 \phi_{f}+2 \phi_{s}\right) .
$$

For the merging of bunches on a porch below transition we have $\phi_{s}=0$ and these equations become

$$
A_{1} \sin \phi_{f}-A_{2} \sin 2 \phi_{f}=0, \quad U_{\phi \phi}=-A_{1} \cos \phi_{f}+2 A_{2} \cos 2 \phi_{f} .
$$

Initially $A_{1}=0$ and $A_{2}>0$ on the porch and we have fixed points $\phi_{f}=0$, $\phi_{f}= \pm \pi / 2$, and $\phi_{f}= \pm \pi$. Motion near $\phi_{f}=0$ and $\phi_{f}= \pm \pi$ is unstable; motion near $\phi_{f}= \pm \pi / 2$ is stable. At the end of the merging process $A_{2}=0$ and $A_{1}>0$ and we have fixed points $\phi_{f}=0$ and $\phi_{f}= \pm \pi$. Motion near $\phi_{f}=0$ is now stable; motion near $\phi_{f}= \pm \pi$ remains unstable.

\subsection{The Separatrix}

Let $H_{u}$ be the value of $H$ at the unstable fixed point

$$
\phi=\phi_{u}, \quad W=0 .
$$

Then

$$
H_{u}=U\left(\phi_{u}\right)
$$

and the equation

$$
H(\phi, W)=H_{u}
$$


defines the separatrix. Solving this equation for $W^{2}(\phi)$ we obtain

$$
W^{2}(\phi)=\frac{2}{a}\left\{U\left(\phi_{u}\right)-U(\phi)\right\}
$$

We also have

$$
\frac{d W^{2}}{d \phi}=-\frac{2}{a} \frac{\partial U}{\partial \phi}, \quad \frac{d^{2} W^{2}}{d^{2} \phi}=-\frac{2}{a} \frac{\partial^{2} U}{\partial \phi^{2}}
$$

from which it follows that $W^{2}(\phi)$ reaches at local maximum at each stable fixed point $\phi_{f}$. The area around the stable fixed point and bounded by the separatrix is an RF bucket. The height, $W_{b}$, of the bucket is given by

$$
W_{b}^{2}=\frac{2}{a}\left\{U\left(\phi_{u}\right)-U\left(\phi_{f}\right)\right\}
$$

where $\phi_{f}$ is the stable fixed point.

For the case in which we are below transition with

$$
F(\psi)=A \sin \psi, \quad A>0, \quad 0 \leq \phi_{s}<\pi / 2
$$

we have

$$
\phi_{u}=\pi-2 \phi_{s}
$$

and

$$
U\left(\phi_{u}\right)=A\left\{\left(\pi-2 \phi_{s}\right) \sin \phi_{s}-2 \cos \phi_{s}\right\}
$$

Thus

$$
U\left(\phi_{u}\right)-U(\phi)=A\left\{\left(\pi-2 \phi_{s}-\phi\right) \sin \phi_{s}-\cos \phi_{s}-\cos \left(\phi+\phi_{s}\right)\right\}
$$

and the separatrix is given by

$$
W^{2}(\phi)=\frac{2 A}{a}\left\{\left(\pi-2 \phi_{s}-\phi\right) \sin \phi_{s}-\cos \phi_{s}-\cos \left(\phi+\phi_{s}\right)\right\} .
$$

For the case in which we are below transition with

$$
F(\psi)=A_{1} \sin \psi-A_{2} \sin 2 \psi, \quad A_{1}>0, \quad A_{2}>0, \quad \phi_{s}=0
$$

we have

$$
U(\phi)=A_{1} \cos \phi-\frac{1}{2} A_{2} \cos 2 \phi-A_{1}+\frac{1}{2} A_{2}
$$

and

$$
\phi_{u}=\pi, \quad U\left(\phi_{u}\right)=-2 A_{1} .
$$

Thus

$$
U\left(\phi_{u}\right)-U(\phi)=-A_{1}\{1+\cos \phi\}-\frac{1}{2} A_{2}\{1-\cos 2 \phi\}
$$

and the separatrix is given by

$$
W^{2}(\phi)=-\frac{2 A_{1}}{a}\{1+\cos \phi\}-\frac{A_{2}}{a}\{1-\cos 2 \phi\}
$$




\section{References}

[1] L.A. Ahrens, et al, "The RHIC Injector Accelerator Configurations, and Performance for the 2003 Au-d Physics Run", Proceedings of the 2003 Particle Accelerator Conference, pp. 1715-1717.

[2] C.J. Gardner, "Kicker Pulse Width Requirements for the Injection of Gold Ions into AGS", C-A/AP/Note 104, August 2003.

[3] K.L. Zeno, "Overview of the Year $2000 \mathrm{Au}^{32+}$ Booster Run", C-A/AP Note 26, October, 2000.

[4] C.J. Gardner, "RF Capture and Acceleration of Gold Ions in Booster", C-A/AP Note 7, November, 1999.

[5] C.J. Gardner, "RF Capture and Acceleration of Gold Ions in Booster-II", C-A/AP Note 28, October, 2000.

[6] J.A. MacLachlan, "Difference Equations for Longitudinal Motion in a Synchrotron", Fermilab internal report FNAL FN-529, December 15, 1989.

[7] J.A. MacLachlan, "Differential Equations for Longitudinal Motion in a Synchrotron", Fermilab internal report FNAL FN-532, January 25, 1990.

[8] H. Yoshida, Numerical Integration Methods, Handbook of Accelerator Physics and Engineering, Edited by A.W. Chao and M. Tigner, World Scientific, 1999, pp. 85-87. 


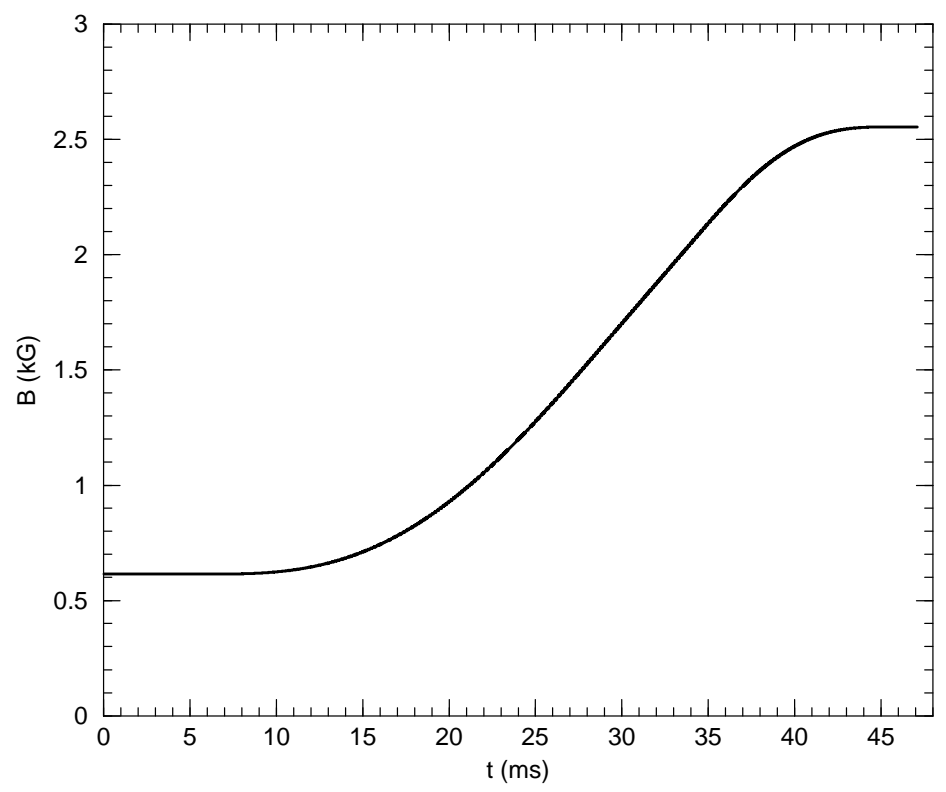

Figure 1: B Field for Acceleration of Gold onto Merging Porch

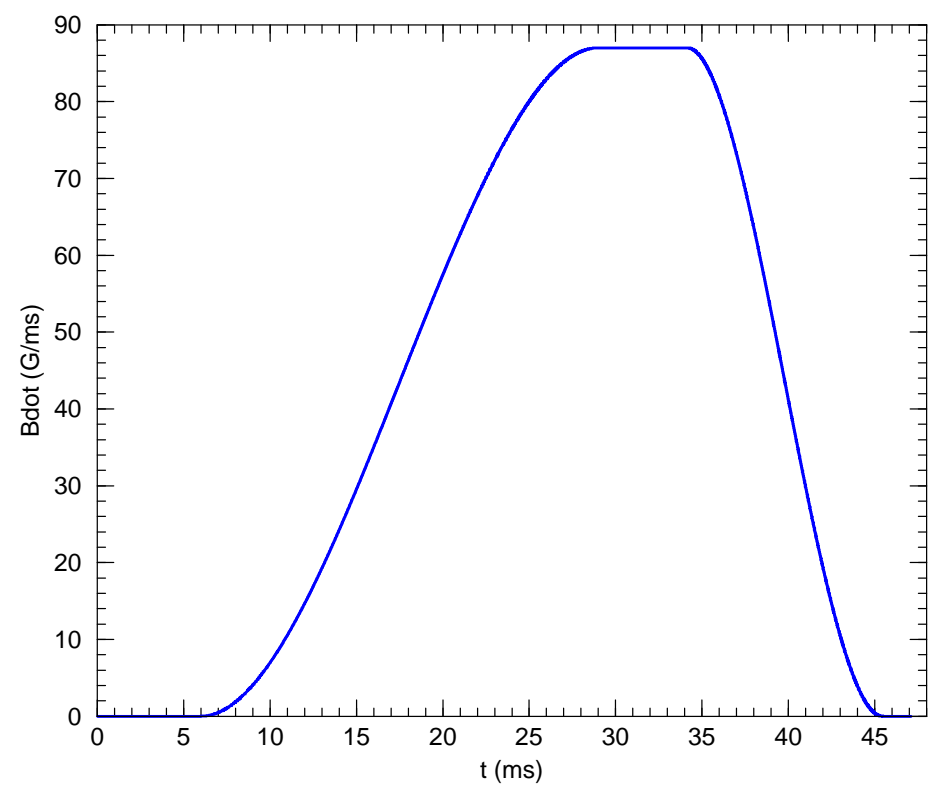

Figure 2: Bdot for Acceleration of Gold onto Merging Porch 


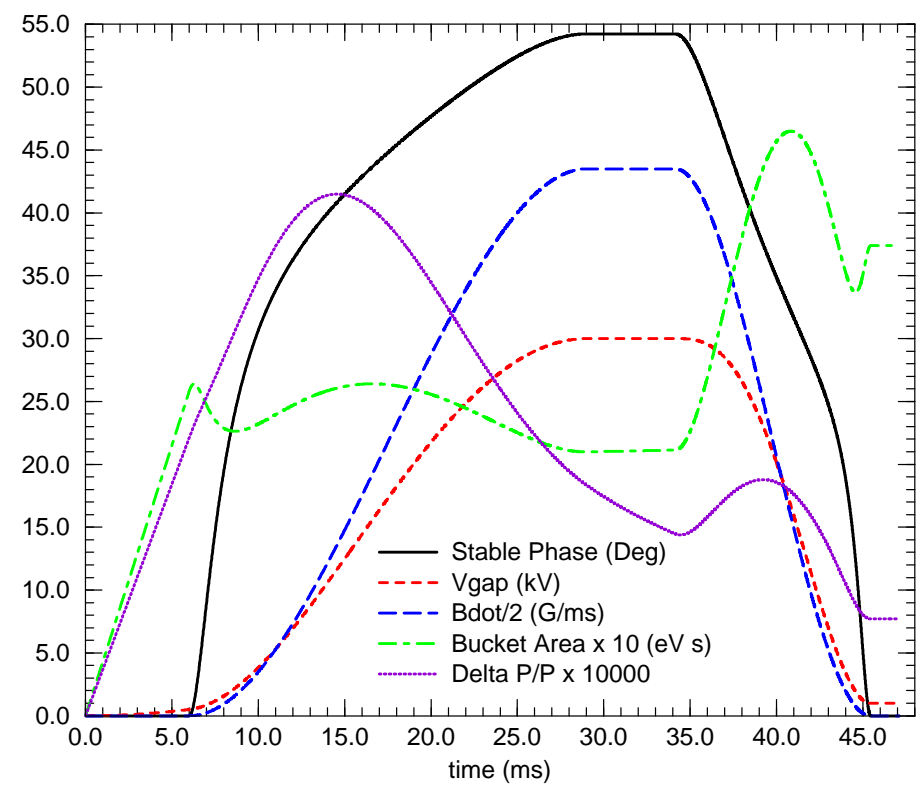

Figure 3: Parameters for Acceleration of Gold onto Merging Porch

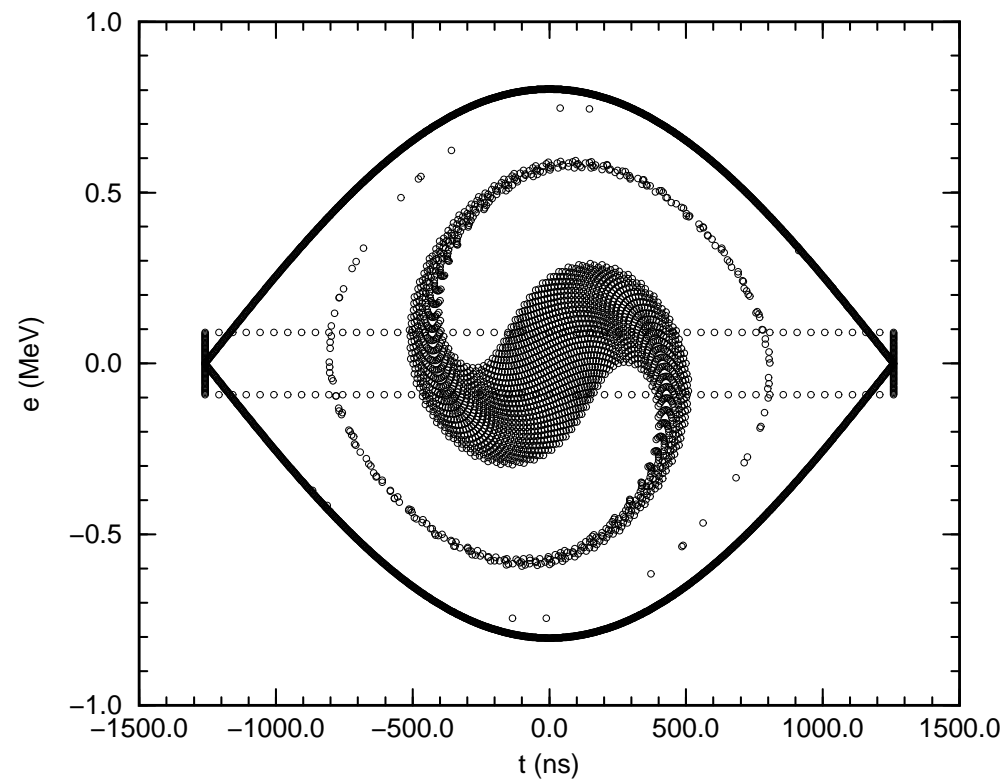

Figure 4: Gold Distribution in RF Bucket at End of Capture 


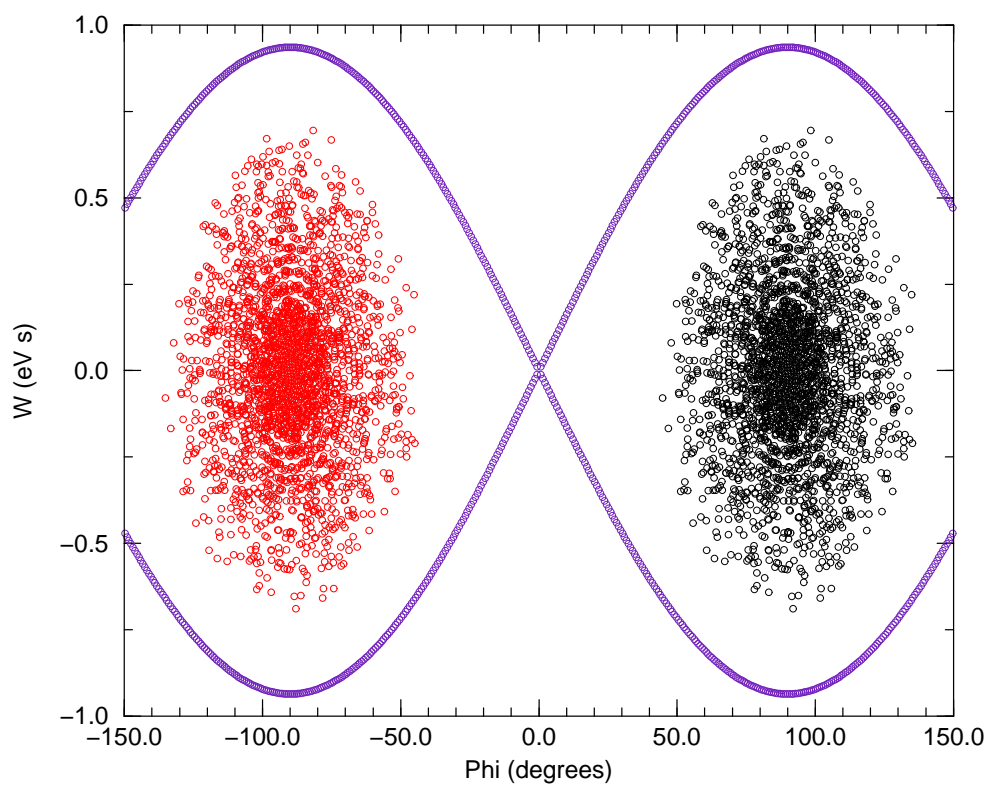

Figure 5: Initial Gold Bunches to be Merged

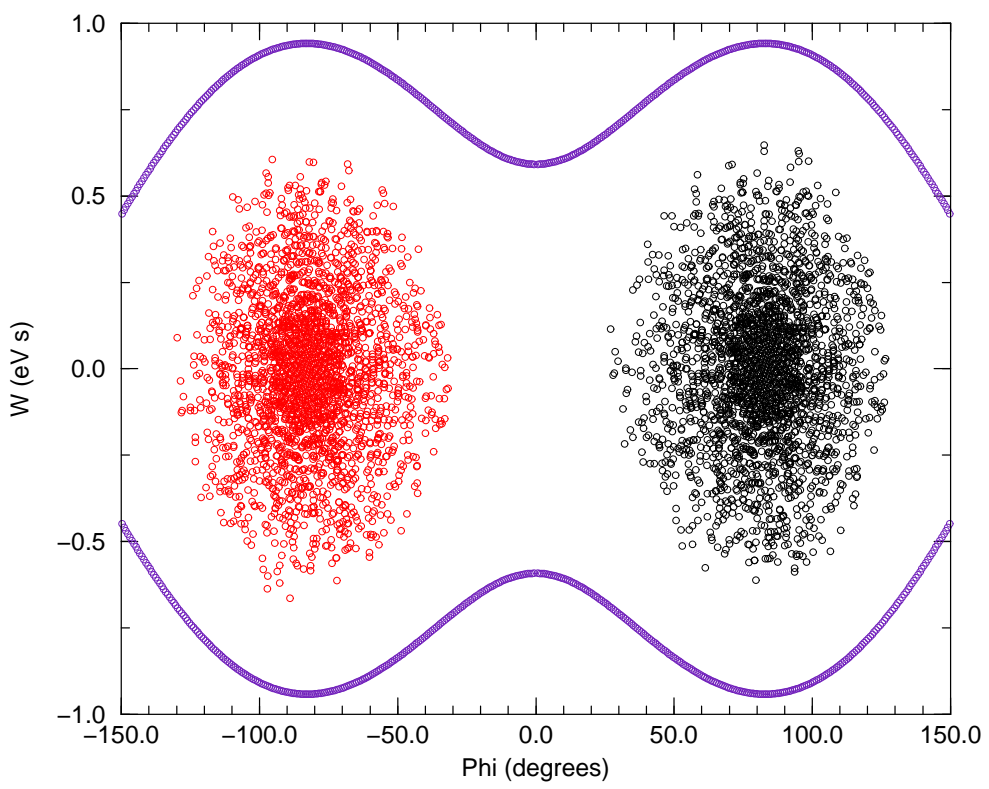

Figure 6: Merge 20\% Complete 


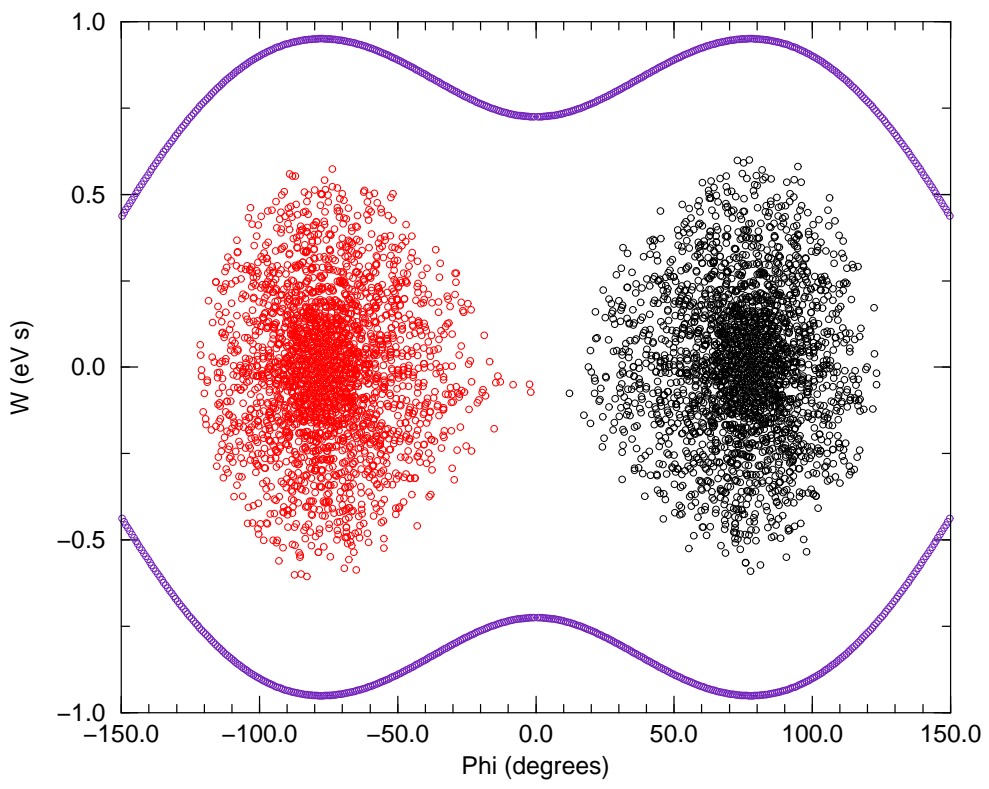

Figure 7: Merge 30\% Complete

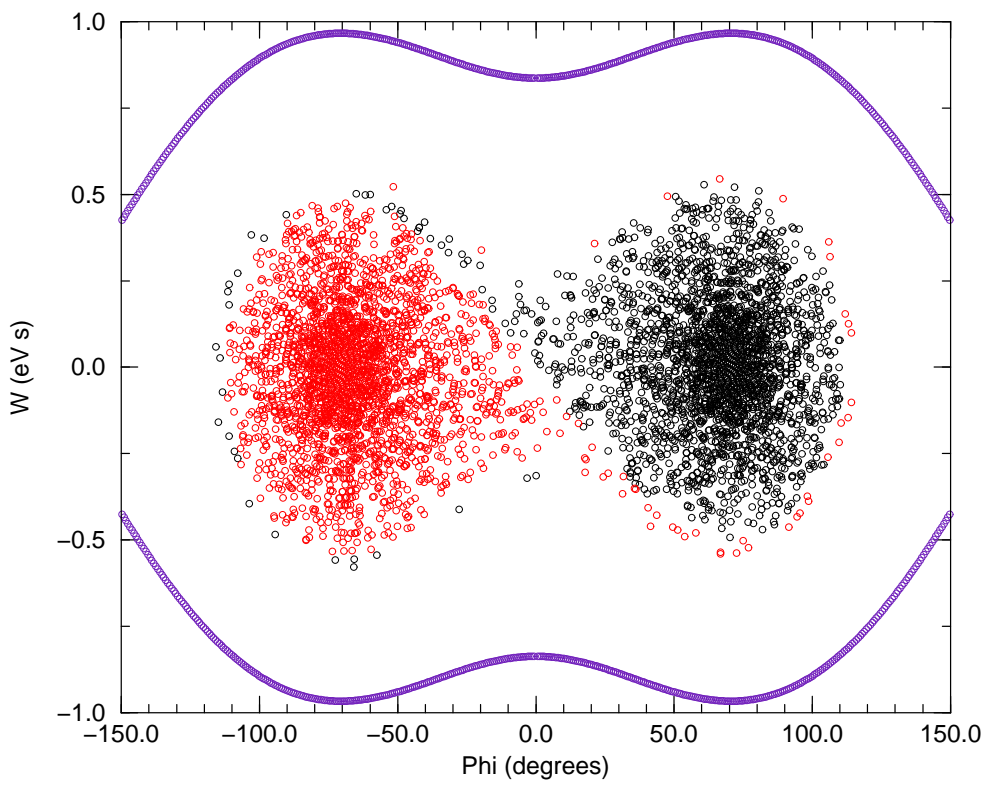

Figure 8: Merge 40\% Complete 


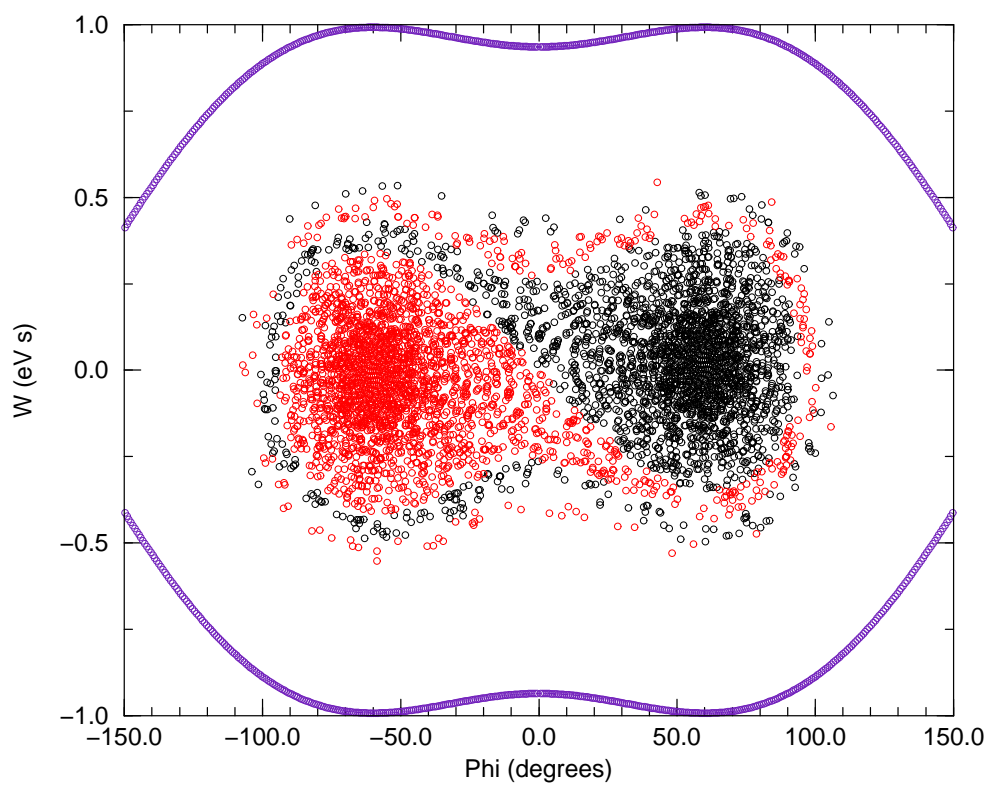

Figure 9: Merge 50\% Complete

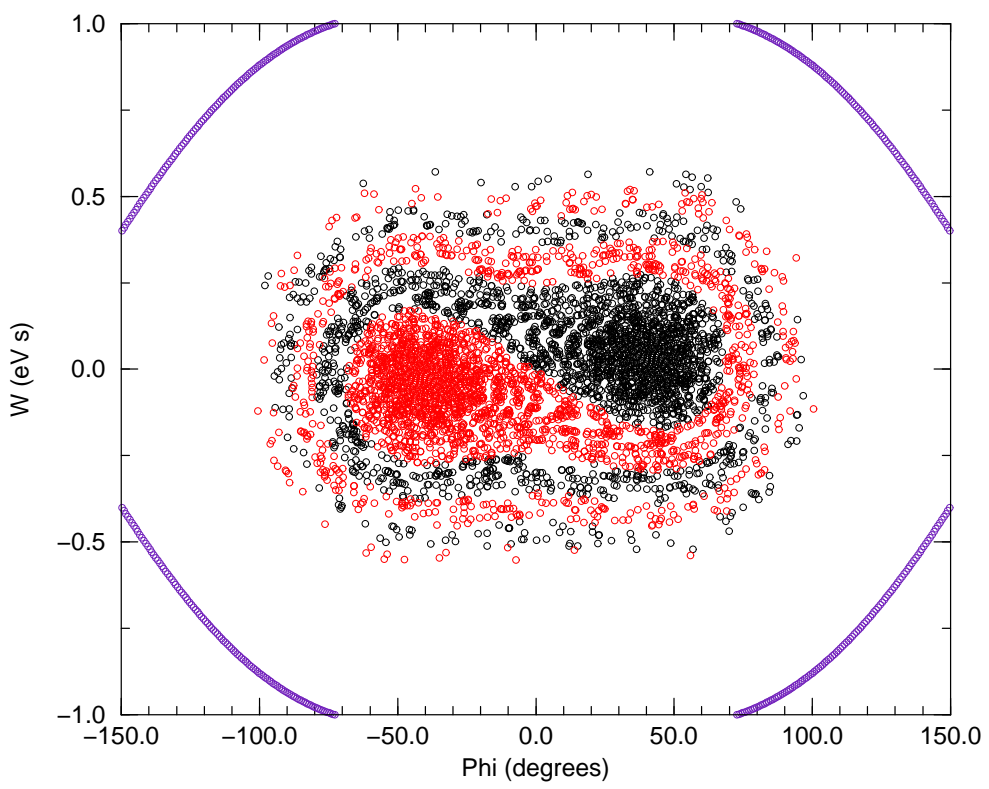

Figure 10: Merge 60\% Complete 


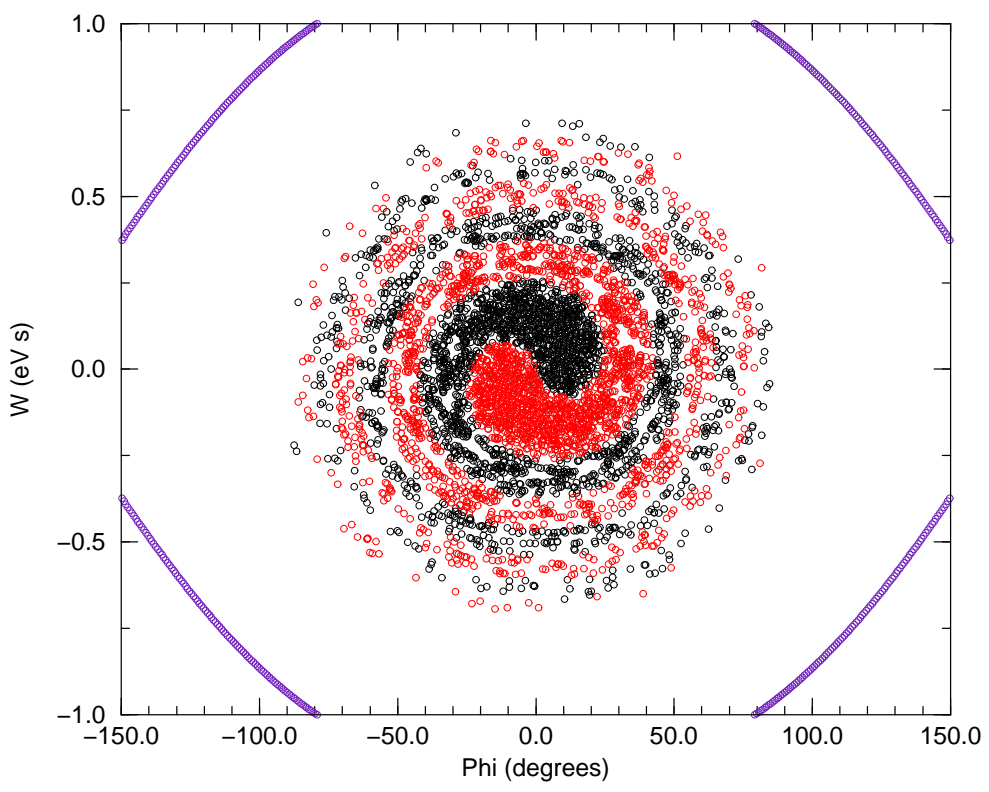

Figure 11: Merge 80\% Complete

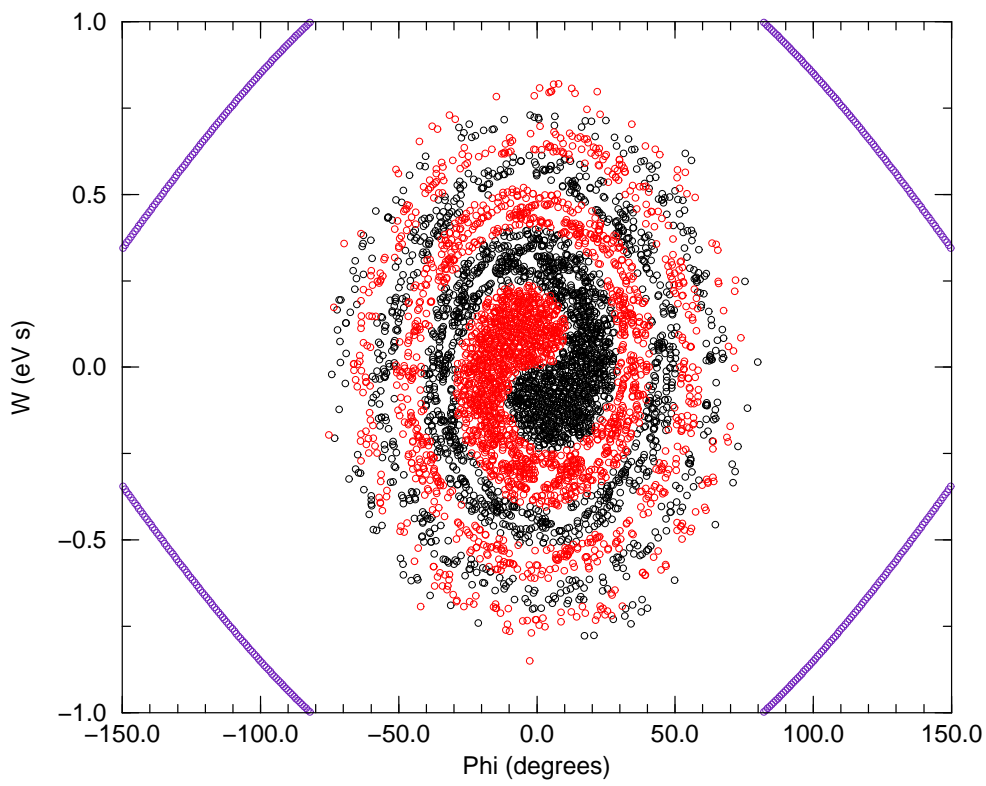

Figure 12: Merge 100\% Complete. Total merging time $40 \mathrm{~ms}$. 


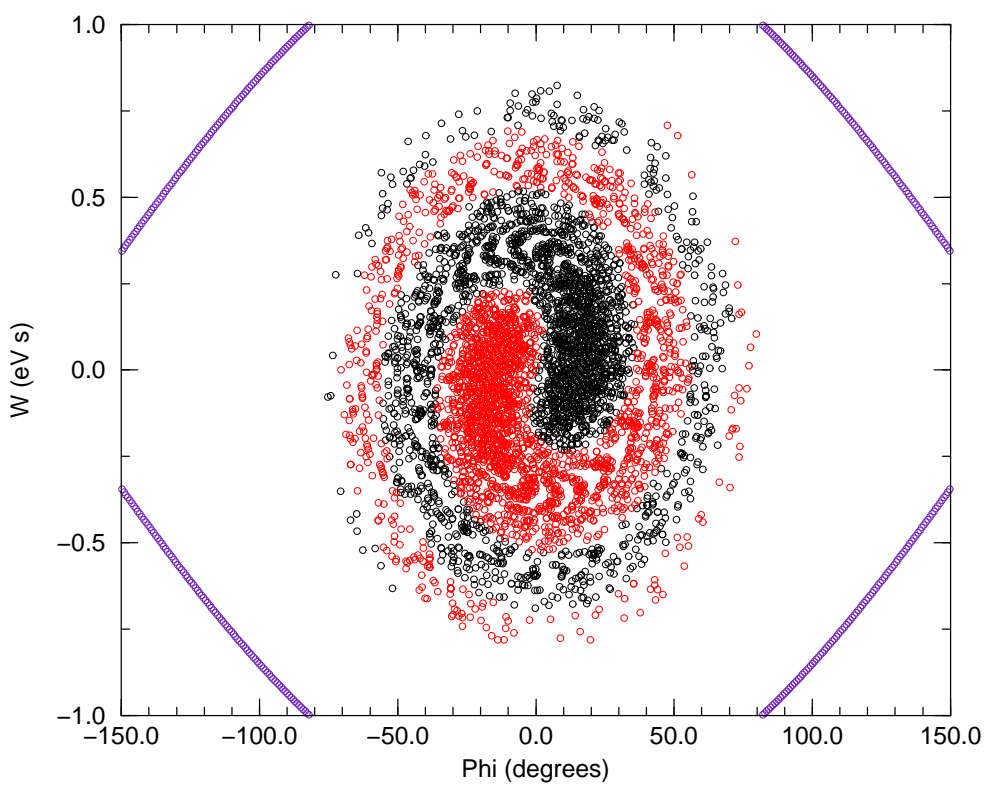

Figure 13: Total merging time $20 \mathrm{~ms}$.

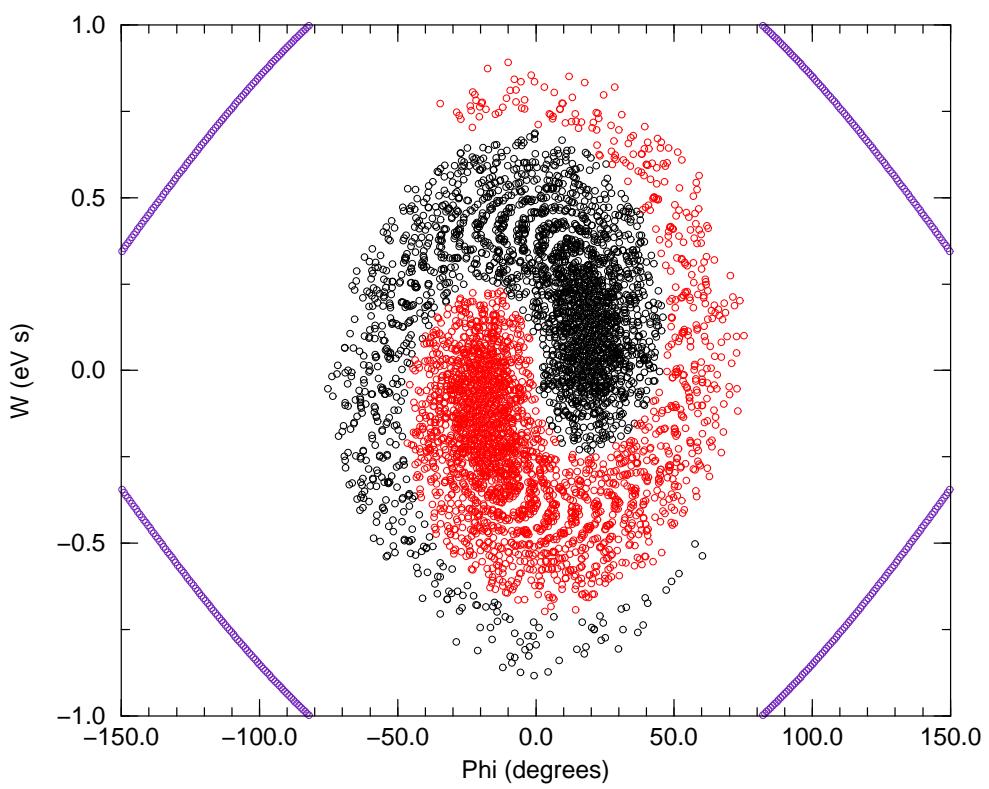

Figure 14: Total merging time $10 \mathrm{~ms}$. 


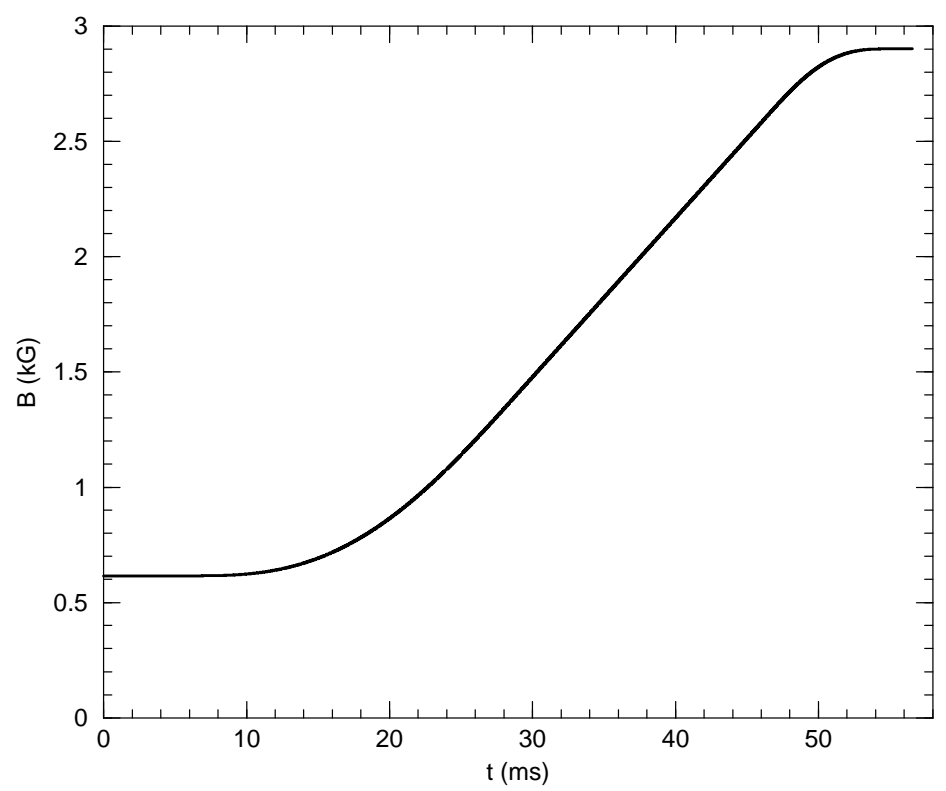

Figure 15: B Field for Acceleration of Deuterons onto Merging Porch

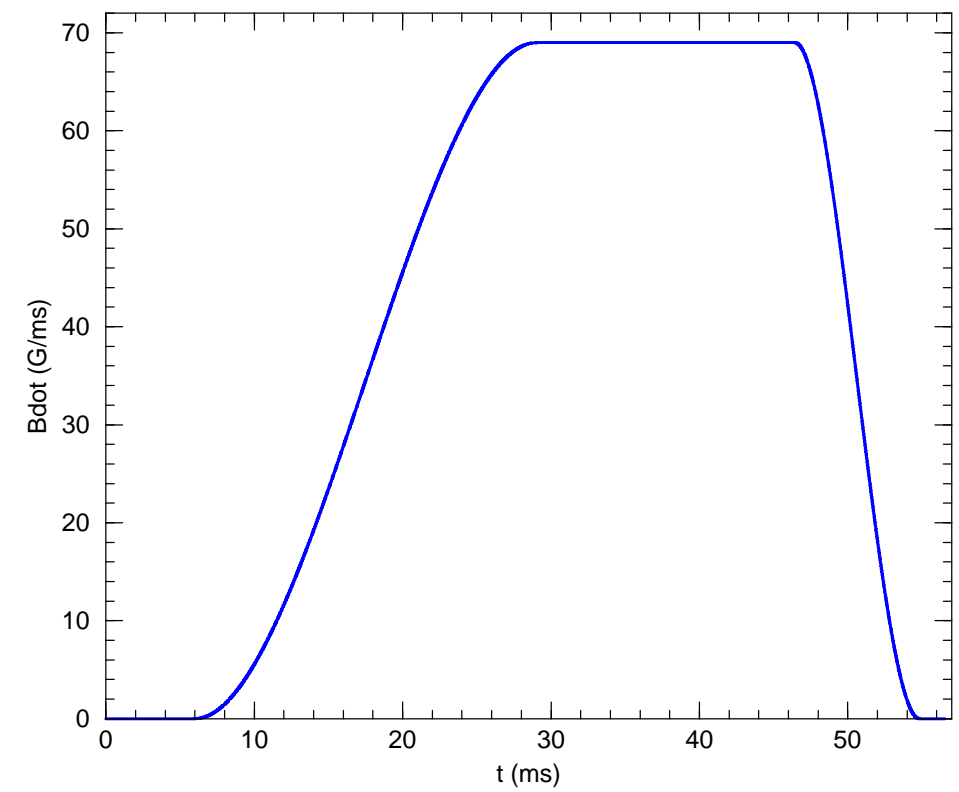

Figure 16: Bdot for Acceleration of Deuterons onto Merging Porch 


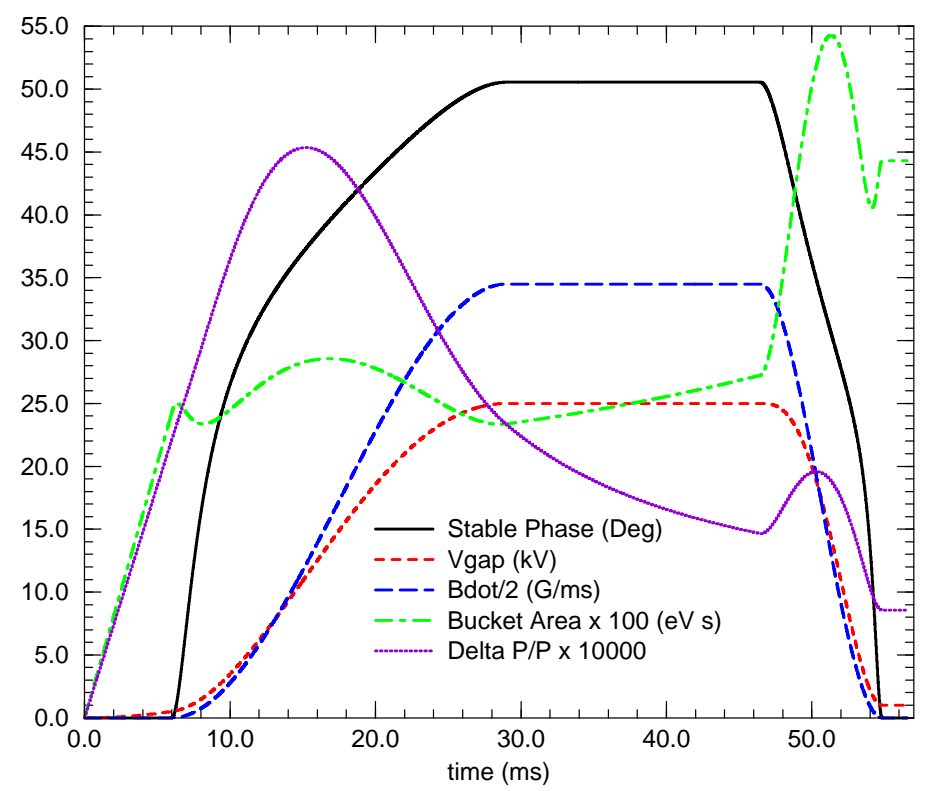

Figure 17: Parameters for Acceleration of Deuterons onto Merging Porch

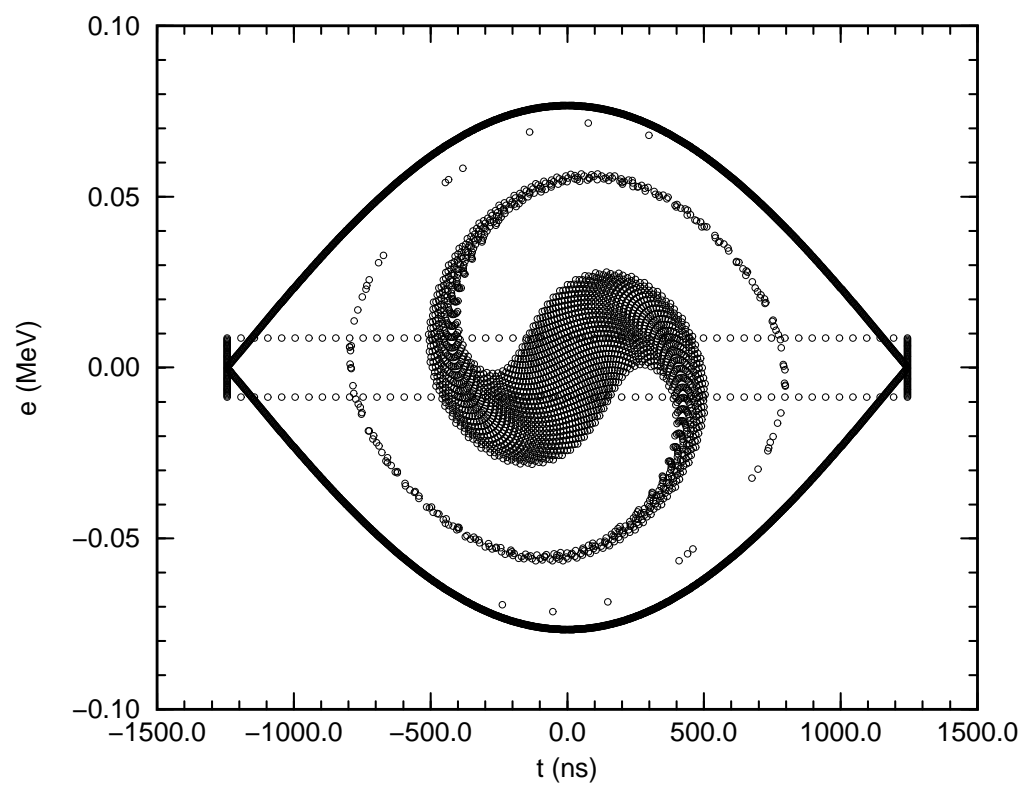

Figure 18: Deuteron Distribution in RF Bucket at End of Capture 


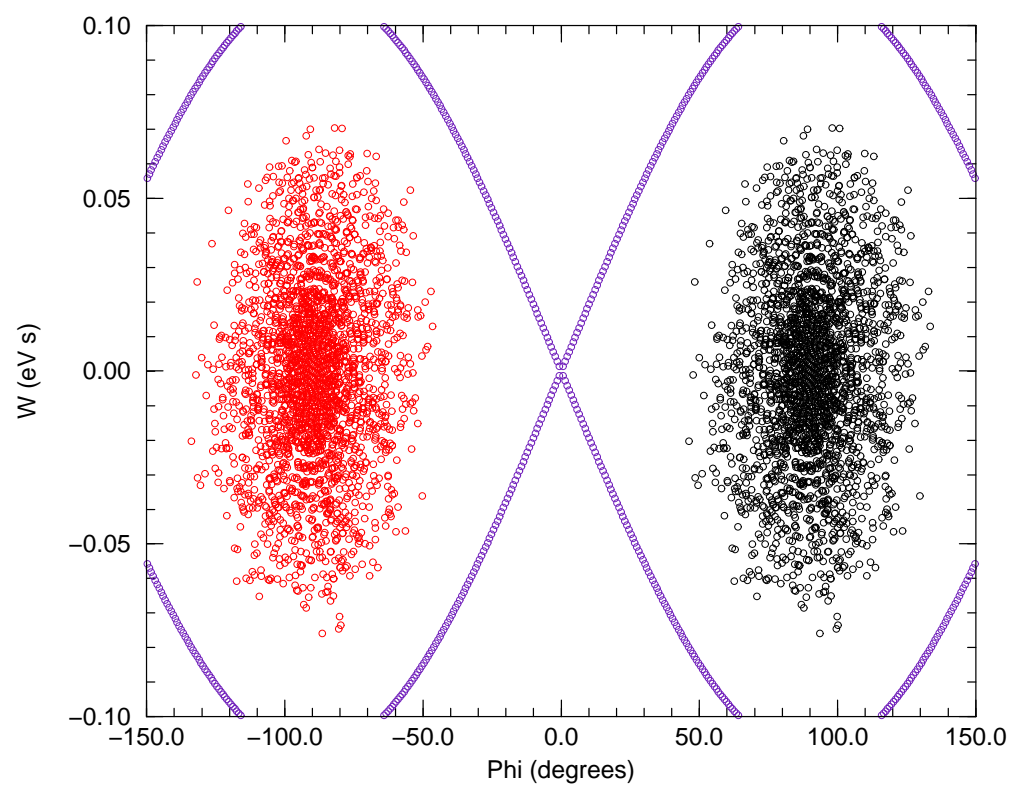

Figure 19: Initial Deuteron Bunches to be Merged

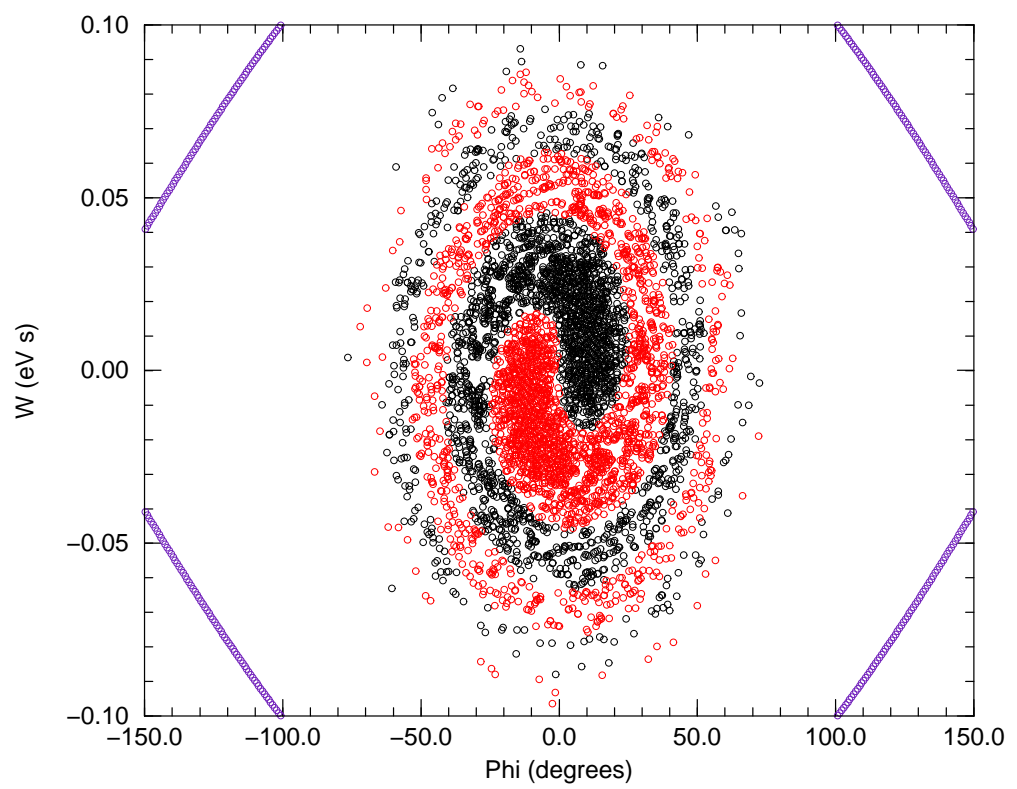

Figure 20: Total Merging Time $40 \mathrm{~ms}$ 


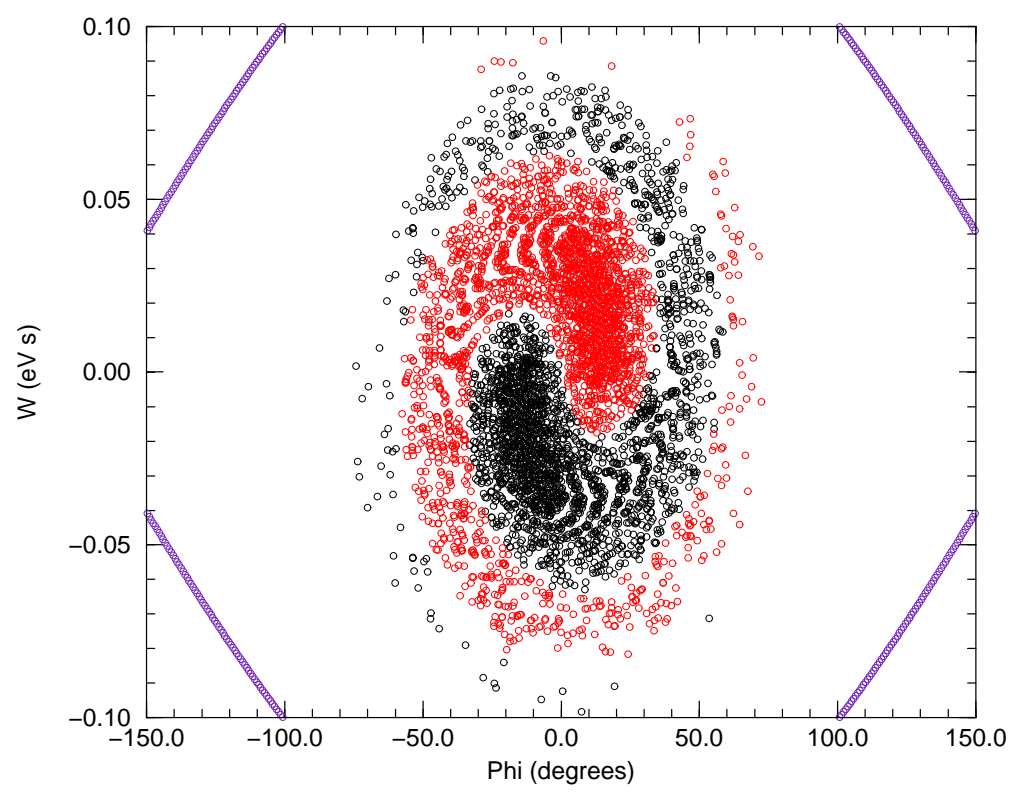

Figure 21: Total Merging Time $20 \mathrm{~ms}$

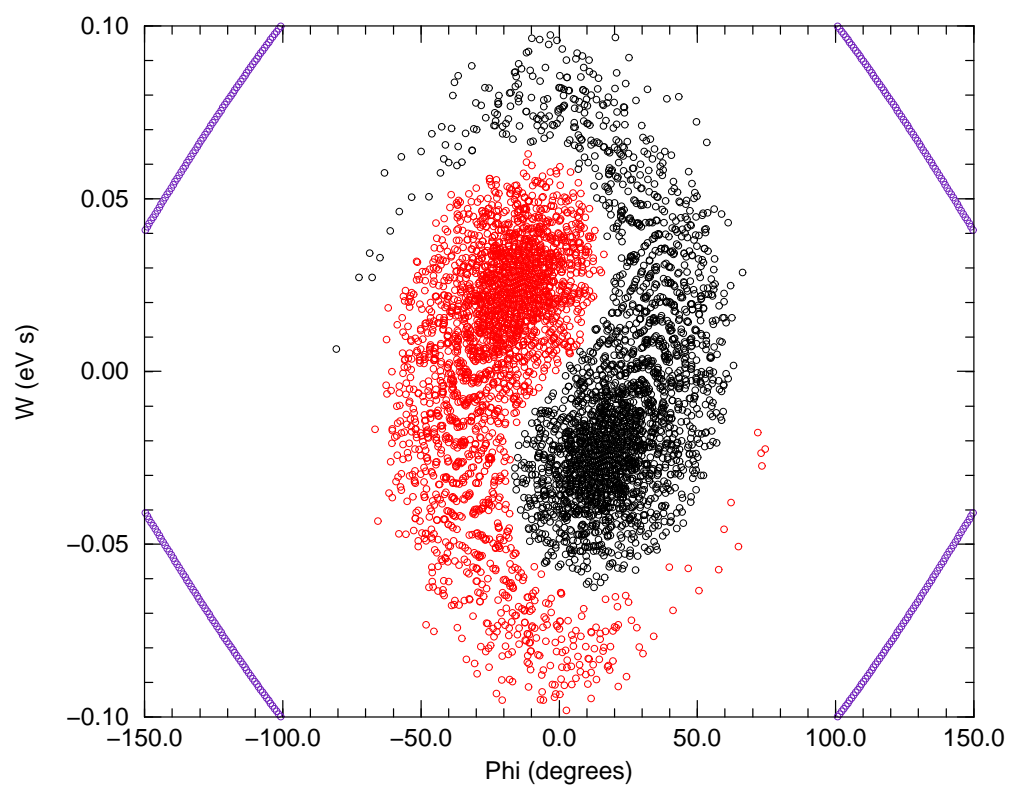

Figure 22: Total Merging Time $10 \mathrm{~ms}$ 